\title{
cGAS-mediated control of blood-stage malaria promotes Plasmodium-specific germinal center responses
}

\author{
William O. Hahn, ${ }^{1,2}$ Noah S. Butler, ${ }^{3}$ Scott E. Lindner, ${ }^{4}$ Holly M. Akilesh, ${ }^{5,6}$ D. Noah Sather, ${ }^{4}$ \\ Stefan H.I. Kappe, ${ }^{4,7}$ Jessica A. Hamerman,, ${ }^{5,8}$ Michael Gale Jr., ${ }^{2,8}$ W. Conrad Liles,, \\ and Marion Pepper ${ }^{2,8}$ \\ 'Division of Allergy and Infectious Diseases and ' Center For Innate Immunity and Immune Disease, University of \\ Washington, Seattle, Washington, USA. ${ }^{3}$ Department of Microbiology, The University of lowa, lowa City, lowa, USA. \\ ${ }^{4}$ Center for Infectious Disease Research, Seattle, Washington, USA. ${ }^{5}$ Immunology Program, Benaroya Research Institute \\ at Virginia Mason, Seattle, Washington, USA. ${ }^{6}$ Division of Rheumatology, Department of Medicine, and 'Department of \\ Clobal Health and ${ }^{8}$ Department of Immunology, University of Washington, Seattle, Washington, USA.
}

Sensing of pathogens by host pattern recognition receptors is essential for activating the immune response during infection. We used a nonlethal murine model of malaria (Plasmodium yoelii $17 X N L)$ to assess the contribution of the pattern recognition receptor cyclic GMP-AMP synthase (cCAS) to the development of humoral immunity. Despite previous reports suggesting a critical, intrinsic role for cGAS in early B cell responses, cCAS-deficient ( $\mathrm{CGAS}^{-/}$) mice had no defect in the early expansion or differentiation of Plasmodium-specific B cells. As the infection proceeded, however, $\mathrm{CCAS}^{-/-}$mice exhibited higher parasite burdens and aberrant germinal center and memory $B$ cell formation when compared with littermate controls. Antimalarial drugs were used to further demonstrate that the disrupted humoral response was not B cell intrinsic but instead was a secondary effect of a loss of parasite control. These findings therefore demonstrate that cGAS-mediated innate-sensing contributes to parasite control but is not intrinsically required for the development of humoral immunity. Our findings highlight the need to consider the indirect effects of pathogen burden in investigations examining how the innate immune system affects the adaptive immune response.

Conflict of interest: The authors have declared that no conflict of interest exists.

Submitted: March 22, 2017 Accepted: November 28, 2017 Published: January 11, 2018

Reference information: JCI Insight. 2018;3(1):e94142. https:// doi.org/10.1172/jci.insight.94142.

\section{Introduction}

Malaria is a disease caused by parasites of the Plasmodium genus that kills approximately 430,000 persons per year (1). The humoral immune response is critical for both acute clearance of blood-stage malaria and protection against subsequent rechallenge (2), yet poor understanding of how to achieve protective humoral immunity hampers vaccine design. The immune response to malaria is initiated when malaria-associated pathogen-associated molecular patterns are recognized by host innate cells via pattern recognition receptors (PRRs) (3). Activation of PRRs has at least two roles in host immunity during blood-stage malaria infection: (a) direct control of parasite replication and/or parasite killing via innate immune effector mechanisms and (b) generation of cues that expand and differentiate antigen-specific CD4 ${ }^{+} \mathrm{T}$ cells and $\mathrm{B}$ cells (3-5). It was recently reported that the PRR cyclic GMP-AMP synthase (cGAS) was a critical innate signal in the context of a murine model of lethal malaria (6). We used a nonlethal murine model of blood-stage malaria (Plasmodium yoelii $17 X N L$ ) to determine the role that cGAS plays in the generation of the humoral immune response and, specifically, immunologic memory.

cGAS is a PRR that senses the presence of cytosolic DNA (7-9); it is important for initiating the host immune response to intracellular bacteria (10-12) and DNA viruses (13). After binding to cytosolic DNA, cGAS enzymatically generates cyclic dinucleotides (2'3' cyclic GMP-AMP) that signal via the adaptor molecule stimulator of interferon genes (STING), with the signaling cascade thought to culminate primarily in the production of type I IFN $(7,8,14)$. While cGAS has been demonstrated to affect $\mathrm{T}$ cell-independent $\mathrm{B}$ cell responses (15), the role of cGAS in driving the humoral response during infection is poorly understood. We therefore hypothesized that disruption of cGAS-mediated immune 
activation could lead to enhanced disease in a nonlethal model of blood-stage malaria.

We therefore utilized the $P$. yoelii $17 X N L$ parasite to examine the differentiation of Plasmodium-specific $\mathrm{CD}^{+} \mathrm{T}$ cells and $\mathrm{B}$ cells in mice with and without cGAS during blood-stage infection. To examine antigen-specific $\mathrm{CD}^{+} \mathrm{T}$ cells responding to infection, $P$. yoelii were generated that constitutively express the Lymphocytic choriomeningitis virus-derived (LCMV-derived) glycoprotein (GP) epitope (GP ${ }_{61-80}$ ). This allows for the identification and analysis of antigen-specific CD4 ${ }^{+} \mathrm{T}$ cells using previously described GP66:I- $\mathrm{A}^{\mathrm{B}}$ tetramer enrichment strategies (16). B cell tetramers were additionally used to identify polyclonal $P$. yoelii-specific B cells (17). Using these tools, we demonstrated that cGAS deficiency had no effect on the early priming, differentiation, or expansion of Plasmodium-specific $\mathrm{CD}^{+} \mathrm{T}$ cells or B cells. We further observed that the absence of cGAS led to poor parasite control, suggesting an as yet unidentified cGAS-dependent mechanism of parasite control. Loss of parasite control was associated with poor germinal center (GC) responses, that could be rectified by pharmacologic clearance of the parasite, further supporting an indirect effect of ongoing parasitemia on the GC response. If parasite replication is controlled, however, cGAS is dispensable for the development of the humoral response. We conclude that manipulation of the cGAS pathway could be pursued as a therapeutic intervention to control parasitemia.

\section{Results}

cGAS-STING-type I Interferon signals contribute to control of parasite burden during blood-stage malaria. To clarify the potential role of the cGAS-STING-type I IFN axis in non lethal blood-stage malaria, we infected $c G A S^{+/+}$or $c G A S^{-/-}$littermate control mice with $P$. yoelii $17 X N L$ infected erythrocytes and measured parasitemia daily via flow cytometry (18). As expected with $P$. yoelii, most infected erythrocytes were CD71 reticulocytes (19) (Figure 1A). Parasitemia reached a peak of approximately $20 \%$ infected erythrocytes at approximately 15 days after infection in $c G A S^{+/+} P$. yoelii-infected mice and was cleared 18-21 days after infection (20). In $c G A S^{-1}$, mice there was an approximately 2-fold increase in parasitemia starting 7 days after infection that persisted until parasites were cleared in both groups around day 22 (Figure 1B). Higher parasite burden in $c G A S^{-/}$mice was associated with worsened weight loss, increased anemia, and poor thermoregulation when compared with littermate controls (Supplemental Figure 1; supplemental material available online with this article; https://doi.org/10.1172/jci.insight.94142DS1). We additionally recapitulated results reported in a lethal strain of $P$. yoelii YM (6) in which immunopathology driven by cGAS is ameliorated in its absence, leading to enhanced $c G A S^{--}$mouse survival (Supplemental Figure 1D).

To further explore the role of the cGAS-STING-type I IFN axis, we repeated our experiments in $I F N A R^{+/+}$and $I F N A R^{-/-}$littermate controls, observing a similar phenotype of increased parasitemia in IFNAR ${ }^{-/-}$mice (Figure 1C). We also infected STING signaling mutant mice (Tmem173"tgt), and similar to $c G A S^{-1-}$ mice, these mice displayed an elevation in parasitemia starting at day 8 after infection and increased anemia, enhanced weight loss, and poor thermoregulation (W.O. Hahn et al., unpublished observations). Poor control of parasitemia in mice deficient in the cGAS-type I IFN axis demonstrates that this axis is important for the host response to blood-stage malaria.

Deficiency in cGAS is associated with altered type I IFN signature. cGAS has been most strongly linked with the production of type I IFN (7), so we assessed the effects of cGAS on gene programs associated with type I IFN signaling. We measured type I IFN and interferon-stimulated gene (ISG) mRNA expression relative to naive mice on days 4, 7, 9, and 14 in bulk splenocytes. We observed decreased expression (fold change relative to naive) of IFN- $\beta$ in $c G A S^{-1-}$ mice compared with WT mice both days 1 and 7 after infection (Figure 2A). Because we observed IFN- $\beta$ mRNA, it was important to examine downstream candidate ISGs. We selected IRF7, IFIT1, and CXCL10, as they had previously been demonstrated to be transcriptionally upregulated in mice with activated cGAS signaling (21). Decreased expression of IRF7, CXCL10, and IFIT1 was observed in $c G A S^{\llcorner-}$mice as compared with WT mice at day 7 and 9 (Figure 2A). To assess whether differences in ISG expression could be attributed to differences in IFN- $\gamma$ production, we also measured IFN- $\gamma$ protein in the serum by ELISA and IFN- $\gamma$ mRNA expression in total splenocytes and observed no difference between $c G A S^{-1-}$ mice and WT controls at any time point examined (W.O. Hahn, unpublished observations).

We also examined IFN-dependent surface expression of PDCA-1 in CD11b+ DCs (22-24). Flow cytometric analysis of $\mathrm{CD} 11 \mathrm{c}^{+} \mathrm{CD} 11 \mathrm{~b}^{+}$dendritic cells after infection revealed no difference in the two experimental groups at 4 days after infection. By 7 days after infection, there was higher expression of PDCA-1 on $\mathrm{CD} 11 \mathrm{~b}^{+}$dendritic cells in WT mice as compared with $c G A S^{-1-}$ mice (Figure $2 \mathrm{~B}$ and Supplemental Figure 2). To confirm that the mRNA and surface expression of ISG molecules reflected bioactive interferon in the 
A

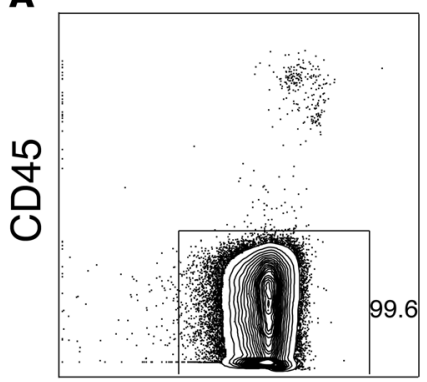

Ter119
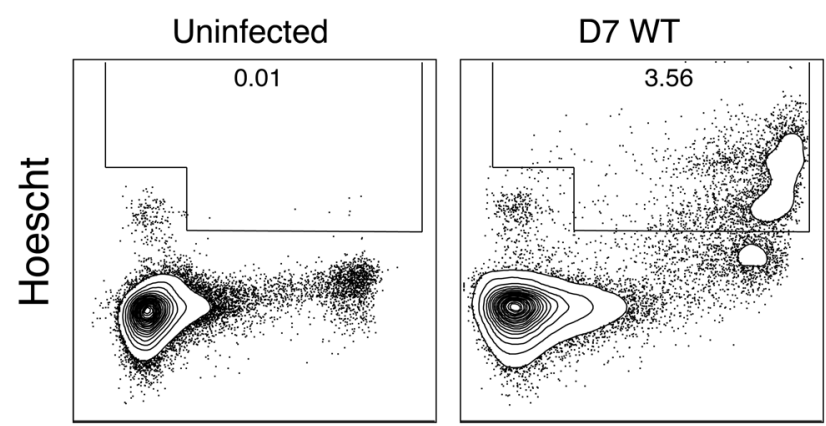

CD71

CD71
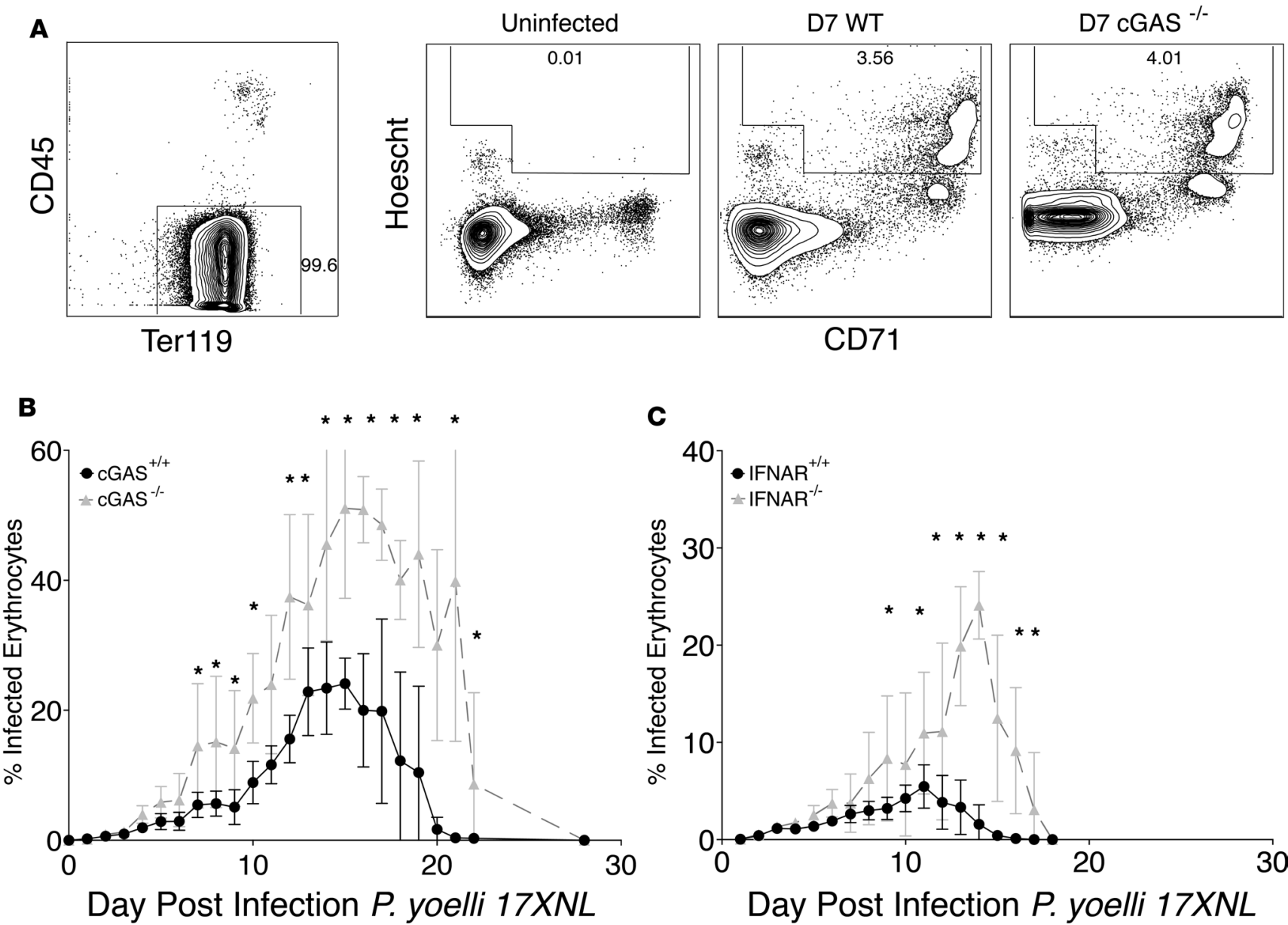

C

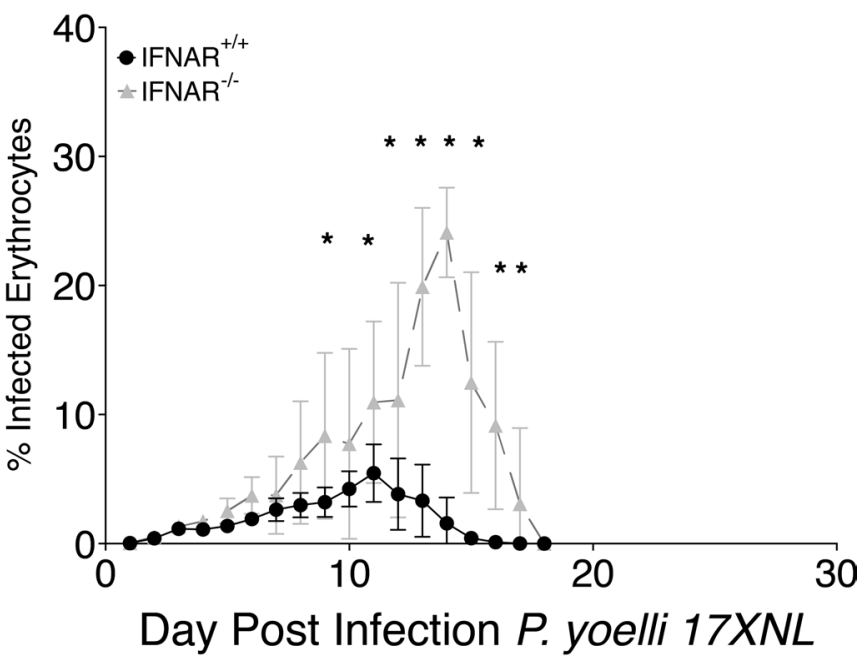

Figure 1. cGAS, STING, and type I interferon contribute to control of blood-stage Plasmodium infection. (A) Flow cytometry gating scheme used to identify infected erythrocytes. Infected erythrocytes were defined as $\mathrm{CD}^{-} 5^{-}$, Ter119+, Hoechst ${ }^{+}$cells. Immature red blood cells (reticulocytes) were identified by expression of CD71. (B) Male $c G A S^{+/+}$and $c G A S^{-/-}$age-matched littermates between 6 and 10 weeks of age were infected with $10^{6}$ Plasmodium yoelii $17 X N L$-infected erythrocytes via the intraperitoneal route. The mean percentage of infected erythrocytes \pm SD is displayed. ${ }^{*} P<0.05$, as assessed by unpaired Student's $t$ test. Each group had at least 4 mice, and infection course was representative of 2 separate experiments. (C) IFNAR ${ }^{+/+}$and IFNAR ${ }^{-/}$ age-matched littermates were infected with $10^{6}$ Plasmodium yoelii $17 X N L$-infected erythrocytes via the intraperitoneal route. The mean percentage of infected erythrocytes \pm SD is displayed. ${ }^{*} P<0.05$, as assessed by unpaired Student's $t$ test. Each group had at least 4 mice, and infection course was representative of 2 separate experiments.

spleen, we utilized an L929 cell line that stably expresses luciferase under a type I IFN-specific promoter (25). Coculture of splenocytes taken from mice at day 7 after infection revealed an elevated luciferase signal in WT mice compared with $c G A S^{-/-}$mice (Figure $2 \mathrm{C}$ ). Together with our IFN- $\beta$ and ISG expression data, these data support a model in which cGAS is required for optimal type I IFN expression.

cGAS deficiency does not affect early antigen-specific $C D 4^{+} T$ cell expansion or differentiation. $C D 4^{+} \mathrm{T}$ cells are critical for the host response to blood-stage Plasmodium infection in addition to being critical for driving T-dependent B cell responses (26). Since our data suggested that there was a defect in the type I IFN cascade and type I IFN signaling has been reported to influence both the expansion and differentiation of $\mathrm{CD}^{+} \mathrm{T}$ cells $(27,28)$, we next sought to determine whether cGAS was associated with defects in early priming or expansion of antigen-specific $\mathrm{CD} 4^{+} \mathrm{T}$ cells during blood-stage malaria infection. To measure antigen-specific $\mathrm{CD} 4^{+} \mathrm{T}$ cell expansion, we utilized a $P$. yoelii parasite modified to stably express amino acids 51-84 of the LCMV GP under the Hep 17 promoter (Supplemental Figure 3A). Expression under the Hep 17 promoter localizes peptide expression to the parasitophorous vacuole, and this promoter is active during the erythrocytic stage of infection (29). This LCMV sequence encodes an immunogenic $\mathrm{CD}^{+} \mathrm{T}$ cell epitope presented on I-A ${ }^{\mathrm{b}} \mathrm{MHC}$ molecules (aa 61-80) $(30,31)$. Infection with $P$. yoelii expressing the $\mathrm{GP}_{66}$ construct drove the expansion of $\mathrm{GP}_{66}$-specific $\mathrm{CD}^{+} \mathrm{T}$ cells that could be identified with a $\mathrm{GP}_{66}: \mathrm{I}^{\mathrm{b}} \mathrm{A}^{\mathrm{b}}$ tetramer (Figure $3 \mathrm{~A}$ ) (32). After infection, $\mathrm{GP}_{66}: \mathrm{I}^{\mathrm{A}} \mathrm{A}^{\mathrm{b}+}$ cells expanded from $328 \pm 132$ 
A

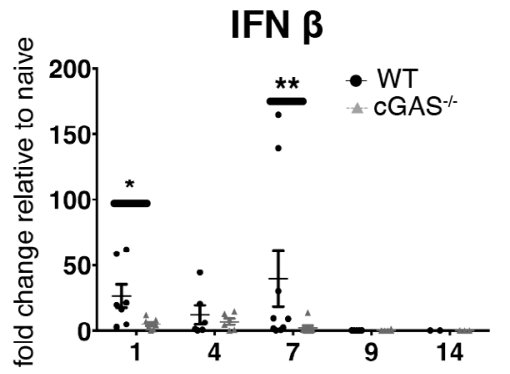

CXCL10

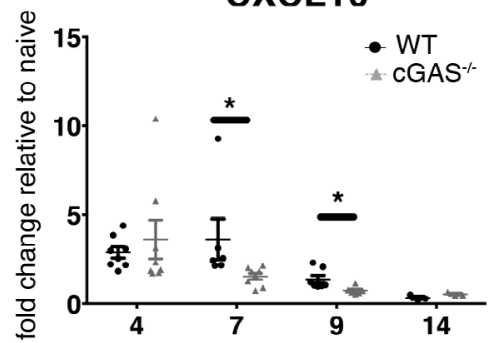

IRF7

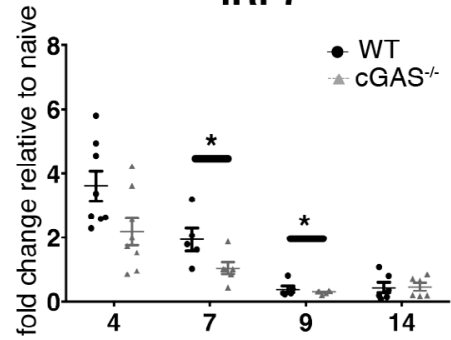

IFIT1

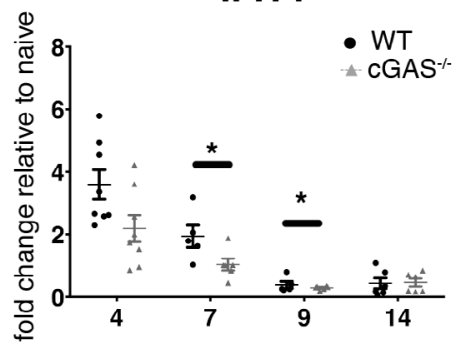

P. yoelli $17 X N L$

B

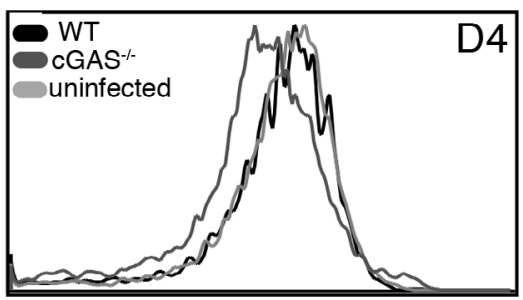

PDCA-1 (CD317)
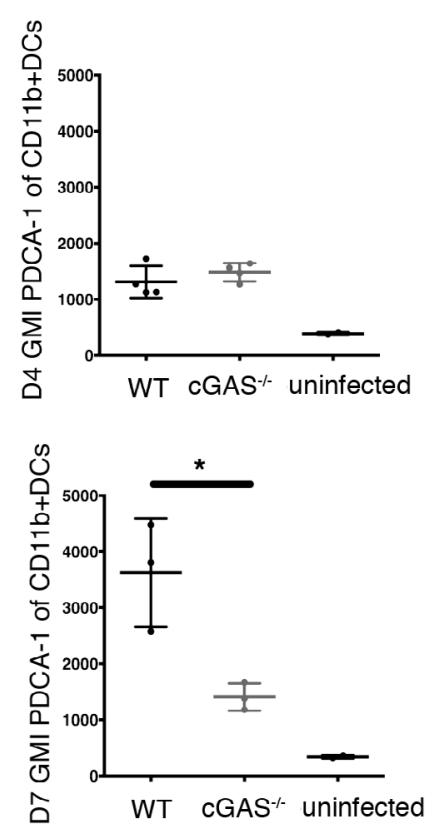

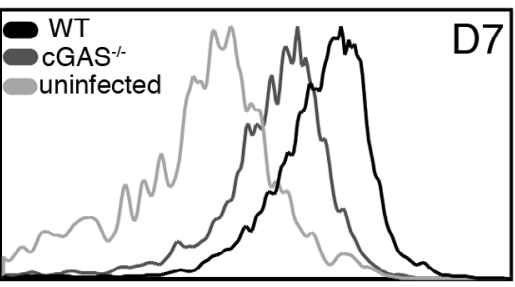

PDCA-1 (CD317)

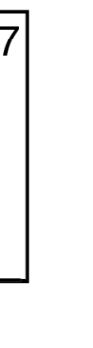

WT cGAS $^{-1-}$ uninfected

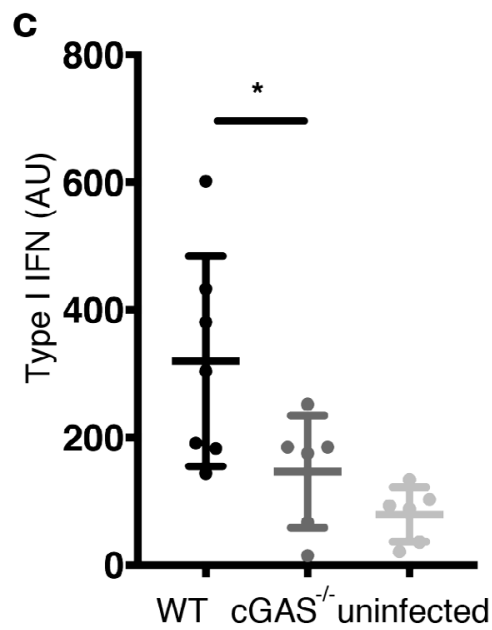

Figure 2. Deficiency in cGAS is associated with altered type I IFN signature. (A) Quantitative real-time PCR of indicated gene mRNA in bulk spleen tissue. Quantification was performed using the delta-delta CT method and normalized to a naive mouse, with HPRT as the designated housekeeping gene.

Experiments were performed using 2 technical replicates of at least 6 biological samples with 2-3 separate experiments per time point. One representative experiment is shown. Since the data were nonparametric, statistical significance was assessed via Mann-Whitney $U$ test. ${ }^{*} P<0.05$, ${ }^{* *} P<0.01$. (B) Mean fluorescent intensity of PDCA-1 (CD317) on CD11 b+ dendritic cells in representative flow plot. See Supplemental Figure 2 for full gating scheme. Representative data are shown. Statistical significance was assessed via Mann-Whitney $U$ test. ${ }^{*} P<0.05$. (C) L929-ISRE cells were plated at $5 \times 10^{4}$ cells per well and cocultured for 6 hours at a ratio of 100 splenocytes to 1 reporter cell in a 96-well plate (mean \pm SD). Cells were lysed and luciferase activity was measured. Statistical analysis was performed using Student's $t$ test. 
cells in a naive mouse to a peak of approximately 40,000 cells by day 9 . We observed no differences in the numbers of $\mathrm{GP}_{66}: \mathrm{I}^{\mathrm{b}+} \mathrm{CD}^{+} \mathrm{T}$ cells in $c G A S^{-1-}$ mice as compared with WT controls on days 4,7 , or 9 after infection (Figure 3A), suggesting that cGAS does not play a role in the priming or expansion of antigen-specific $\mathrm{CD}^{+} \mathrm{T}$ cells during $P$. yoelii infection.

Early type I IFN receptor blockade via antibody has recently been reported to enhance the formation of the total $\mathrm{T}$ follicular helper (Tfh) $\mathrm{CD}^{+} \mathrm{T}$ cell population in $P$. yoelii-infected mice (33), so we sought to determine if an absence of cGAS altered the differentiation of $\mathrm{CD}^{+} \mathrm{T}$ cells responding specifically to infection. As reported for the nonantigen-specific $\mathrm{CD}^{+} \mathrm{T}$ cell response in $P$. chabaudi (34), $\mathrm{GP}_{66}: \mathrm{I}_{-} \mathrm{Ab}^{+} \mathrm{CD} 4^{+}$ T cells formed in response to $P$. yoelii were predominantly CXCR5 ${ }^{+}$Tfh cells, with strong upregulation of CXCR5. By day 7, approximately $80 \%$ of $\mathrm{CD} 44^{+} \mathrm{GP} 66^{+} \mathrm{CD} 4^{+} \mathrm{T}$ cells had upregulated CXCR5 in both $c G A S^{-1-}$ and WT mice (Figure 3B). Furthermore, we observed no differences in the intracellular expression of the Th1 master transcription factor Tbet on $\mathrm{GP}_{66}: \mathrm{I}_{-} \mathrm{A}^{\mathrm{b}+} \mathrm{CD}^{+} \mathrm{T}$ cells in $c G A S^{-1}$ mice as compared with controls on day 7 after infection (data not shown). From these observations, we concluded that cGAS does not affect the early expansion and differentiation of antigen-specific CD4 ${ }^{+} \mathrm{T}$ cells.

cGAS does not affect early antigen-specific B cell expansion or differentiation. Previous investigations have demonstrated that antibodies play a critical role in the host response to acute blood-stage malaria infection with $P$. yoelii $X N L$ (35), so an alternative hypothesis was that cGAS was affecting the early B cell response. Early production of antibodies can occur via a short-lived CD138 $8^{+}$plasmablast B cell subset (36, 37). Because cGAS has been reported to affect the T-independent B cell response (15), we hypothesized that the loss of parasite control we had observed in $c G A S^{-/-}$mice could be associated with a loss of early plasmablast formation in a $\mathrm{T}$ cell-independent fashion. To examine the antigen-specific B cell response, we used a B cell tetramer comprising the C-terminal portion of the merozoite surface protein 1 (MSP1) protein of $P$. yoelii $(17,38)$. Antibodies against MSP1 are associated with clinical immunity in endemic populations $(39,40)$ and murine models of Plasmodium infection $(41,42)$. Furthermore, memory B cells specific for this protein can be found in malaria-exposed individuals living in Plasmodium endemic regions $(43,44)$. To test the role of cGAS in the development of the humoral immune response, the differentiation of MSP1-specific B cells was assessed after $P$. yoelii infection of WT and $c G A S^{-1-}$ mice (Figure $4 \mathrm{~A}$ ). As we found with $\mathrm{CD}^{+} \mathrm{T}$ cells, there were no differences in the expansion of total MSP1-specific B cells at day 7 after infection, a time point at which $\mathrm{CD} 138^{+}$plasmablasts and activated GC precursors can be found (43). The development of MSP1-specific CD138- GL7 ${ }^{+} \mathrm{CD} 38^{+}$activated precursor B cells (45) was also similar in $c G A S^{-1-}$ mice and WT controls 7 days after infection (Figure $4 \mathrm{C}$ ). We also measured the concentration of IgG2c and IgM antibodies in the sera of infected $c G A S^{-/}$and $W T$ mice at day 9 after infection. IgM production has been shown to be diminished in mice lacking cGAS compared with cGAS-sufficient mice exposed to a viral infection (15). IgG2c has been shown to be the predominant isotype formed in response to $P$. yoelii infection (46) and is most strongly associated with protection when administered to naive mice (47). We elected to measure serum antibodies on day 9, after differences in parasite load were observed in $c G A S^{-1-}$ versus $W T$ mice (Figure $1 \mathrm{~B}$ ). We reasoned that if serum antibody levels were similar, this would argue against antibodies contributing significantly to cGAS-mediated parasite control at this time point. Serum concentrations of both isotypes, as measured by ELISA, were not different 9 days after infection (Figure 4D). We also observed no differences in the percentage or number of plasmablasts formed by day 7 (Figure 4, B and E). Therefore, cGAS did not affect the early activation, differentiation, or antibody production of antigen-specific B cells responding to infection with P. yoelii 17XNL.

cGAS deficiency reduces GC formation late in infection. Malaria infection has been associated with impairment of GC formation in both natural infection and model systems. While we had observed no obvious differences in the formation of $\mathrm{CD}^{+} \mathrm{T}$ cell $\mathrm{Tfh}$ cells or activated GC precursor B cells in WT versus $\mathrm{cGAS}^{-/-}$mice, we wanted to examine the effects of persistent infection on GC formation. GC formation was first assessed by histology. We defined a follicle by an area with clear T cell and B cell zones, and we defined PNA-expressing cells centrally located within a follicle as an active GC. On day 14 after infection, WT animals demonstrated a greater percentage of $\mathrm{PNA}^{+}$follicles when compared with cGAS ${ }^{-/-}$mice (Figure $5 \mathrm{~A}$ ). In keeping with a greater percentage of GCs in WT mice compared with $\mathrm{CGAS}^{-/}$mice, we also observed significantly higher concentrations of MSP1-specific IgG2c antibodies on day 14 after infection in WT mice as compared with $\mathrm{CGAS}^{-/-}$mice, with no difference in the IgM response to MSP1 (Figure 5B).

Due to the obvious differences in GC histology and poor formation of antibodies in $\mathrm{CGAS}^{-/-}$mice compared with WT mice, we hypothesized that there could be a defect in the generation of GC CD4 ${ }^{+} \mathrm{Tfh}$ 
A

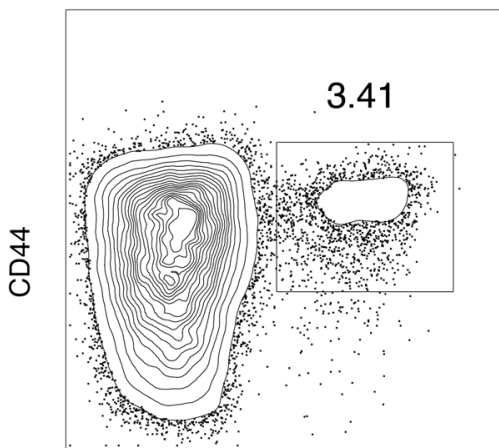

GP66 I-A

B

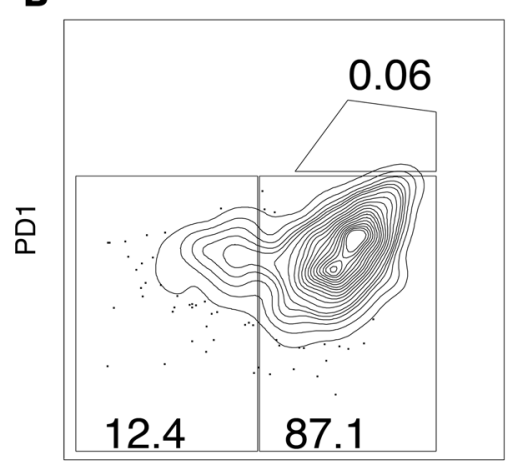

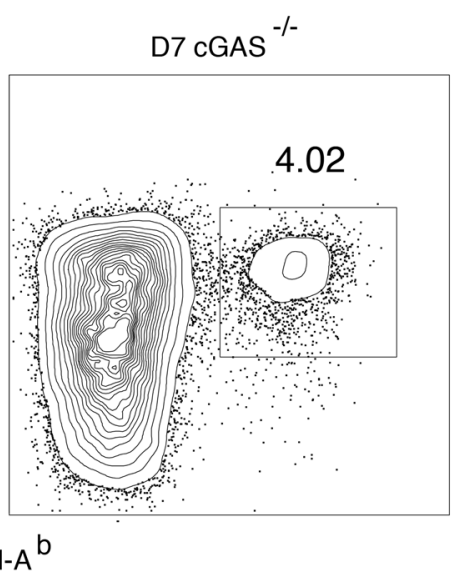

D7 $\mathrm{cGAS}^{-/-}$

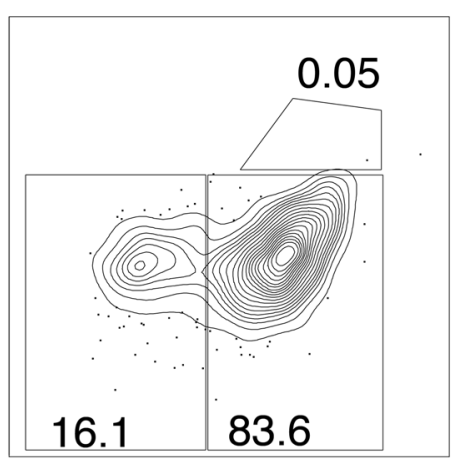

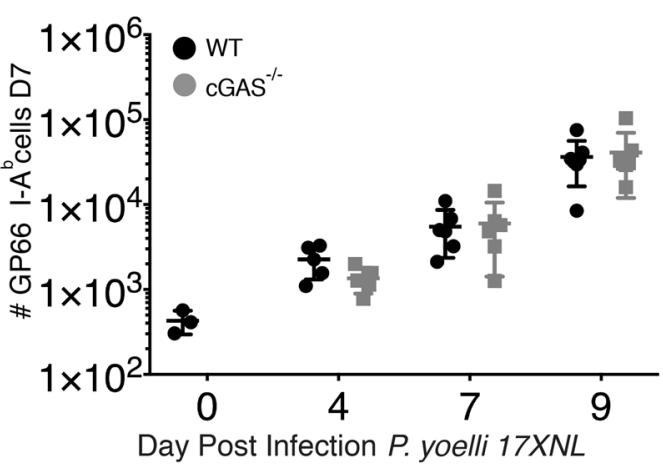

Day Post Infection P. yoelli 17XNL

\section{CXCR5}

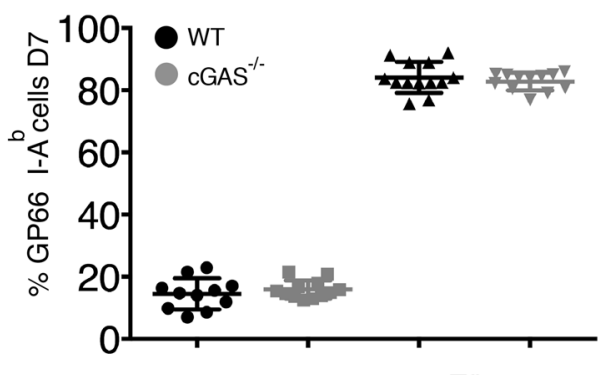

Tef
Tfh

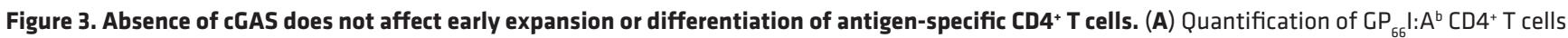
enriched from spleen and lymph nodes on indicated days after infection. Cells were gated on singlets, CD11b/CD11c/B220, CD4+ lymphocytes. Each time point represents data from at least 2 separate experiments with at least 6 mice per time point, except for the day 0 time point, where $n=3$. Error bars represent SD. (B) Representative flow plots demonstrating CXCR5 and PD1 surface expression from CD44 $\mathrm{CP}_{66} \mathrm{I}^{\mathrm{b}+} \mathrm{CD} 4^{+} \mathrm{T}$ cells in the spleen and lymph nodes 7 days after infection with Plasmodium yoelii 17XNL. Data shown are representative of 4 separate experiments with at least 9 biological replicates. There were no statistical differences between any groups. Statistical analysis was performed using the unpaired Student's $t$ test.

(GC Tfh) cells in $\mathrm{cGAS}^{-/-}$mice late in infection. GC Tfh cells can be identified by their high expression of both the B cell-homing chemokine receptor CXCR5 (48) as well as high expression of the inhibitory receptor PD-1 (49). An examination of the GP66:I-Ab+ cells 14 days after infection revealed a reduction of GC Tfh cells in the $\mathrm{CGAS}^{-/-}$mice compared with WT mice associated with a concomitant decrease in the percentage and number of GP66:I-Ab+ $\mathrm{CXCR}^{-} \mathrm{T}$ effector cells (Figure $5 \mathrm{C}$ ). Interestingly, in the absence of cGAS signaling, the majority of GP66:I-Ab ${ }^{+}$cells resembled CXCR5 $5^{\text {int }} \mathrm{PD} 1^{\text {lo }} \mathrm{Tfh}$ cells, thought to localize to either the T-B border or in the extrafollicular region (50).

These Tfh cells have also been associated with the production of plasmablasts in both autoimmune disease and infection (26), so we therefore looked to determine if there were differences in antigen-specific B cell formation. While there were no differences in the absolute number of plasmablasts, $\mathrm{CGAS}^{-/-}$mice still had a small but significant increase in the percentage of $\mathrm{B} 220^{+} \mathrm{CD} 138^{+}$plasmablasts compared with WT controls (Figure 5D and Supplemental Figure 4). Similarly, there was an increased percentage of $\mathrm{CD} 38^{+} \mathrm{GL}^{+} \mathrm{MSP} 1$-specific B cells - markers previously associated with an activated B cell population capable of generating both memory and GC B cells ("activated precursors") of total activated precursors (27) - in $\mathrm{cGAS}^{-/-}$mice compared with WT mice (Figure 4E and Supplemental Figure 4). We also observed a 2 -fold decrease in the number and a decrease of approximately $10 \%$ in the frequency of GC B cells in $\mathrm{cGAS}^{-/-}$mice compared with WT controls (Supplemental Figure 4 and Figure $5 \mathrm{E}$ ). We can therefore conclude from these data that, in mice that lack cGAS, there are fewer antigen-specific GC Tfh cells and GC B cells, a greater percentage of plasmablasts and activated precursor B cells, and reduced antibody titers. 

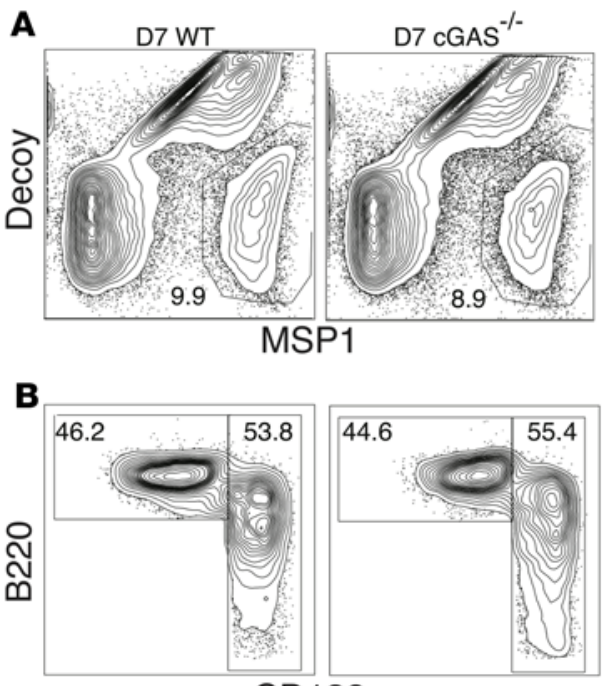

CD138

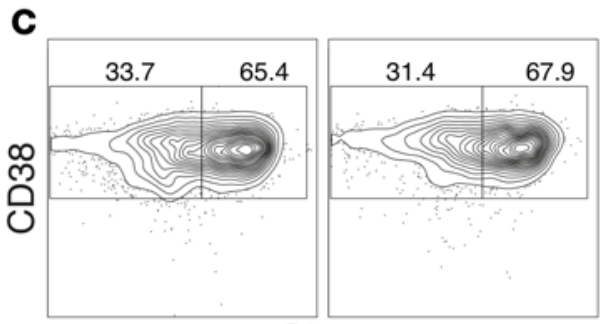

GL7

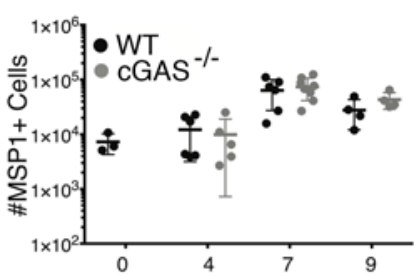

Day Post Infection P. yoelli 17XNL
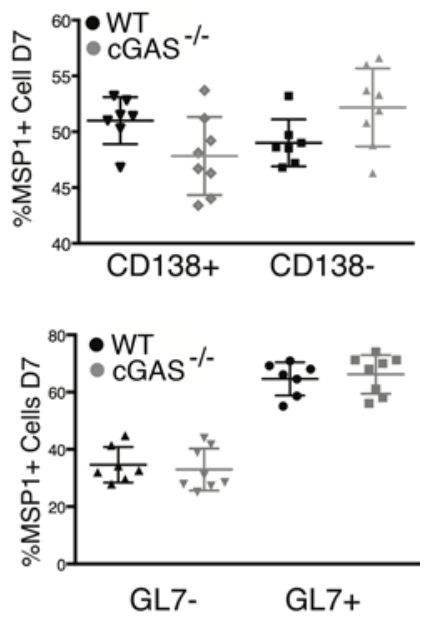

Persistent infection causes GC collapse and reduction in $B$ cell memory. Because we had observed marked differences in the development of GC responses 14 days after infection in $c G A S^{-/}$mice as compared with WT controls, we sought to determine the consequences for the development of cellular memory at a time point at which we had previously found MSP1-specific memory $\mathrm{B}$ cells to be present (38). Immunofluorescent staining of spleens from WT mice demonstrated ongoing $\mathrm{PNA}^{+} \mathrm{GCs}$, whereas there was an absence of $\mathrm{PNA}^{+} \mathrm{GCs}$ in $c G A S^{-/-}$mice (Figure $6 \mathrm{~A})$. We further characterized the $\mathrm{MSP} 1^{+} \mathrm{B}$ cell populations by flow cytometry and determined that not only did $c G A S^{-/-}$mice have fewer total

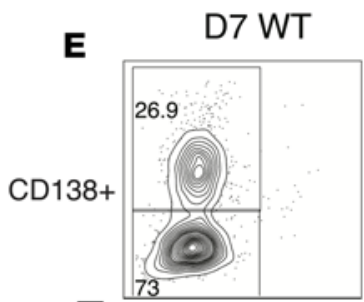

D7 cGAS ${ }^{-\prime}$
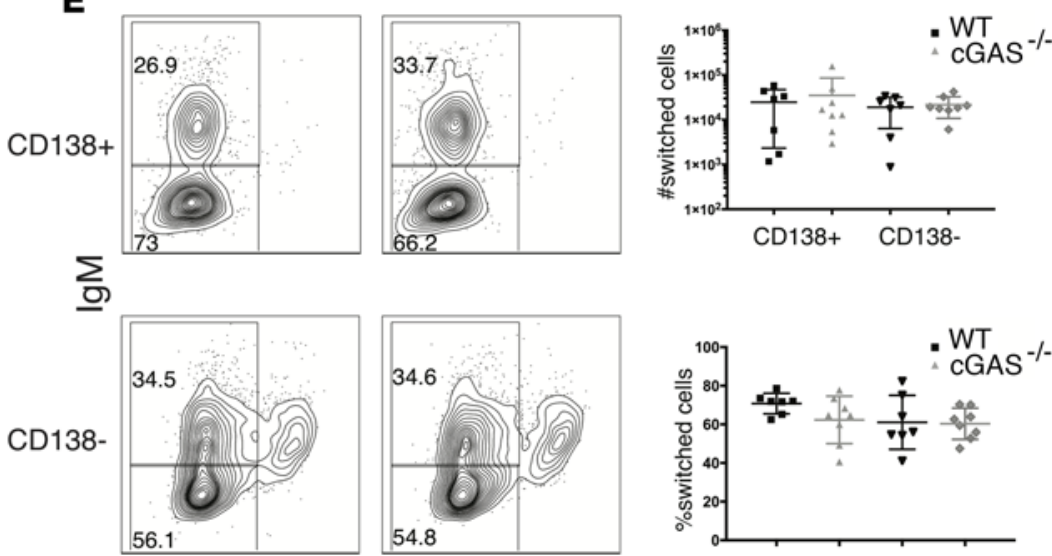

$\lg \mathrm{D}$

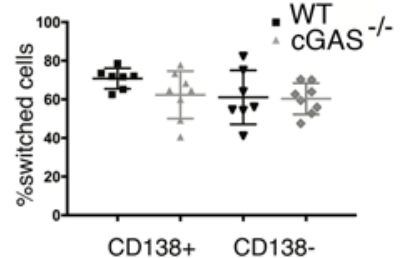

MSP1-specific cells, but more specifically they had fewer GC and memory B cells than WT mice (Figure 6B). Of interest, there were higher numbers of $\mathrm{CD}_{138^{+}}$cells in $c G A S^{-/}$mice than WT mice (Figure 6B). This altered humoral response in $c G A S^{-/-}$mice was also reflected in lower levels of MSP1-specific IgG2C but not IgM (Figure 6, C and D). Expression of CD73, a marker associated with $\mathrm{B}$ cells that have interacted with $\mathrm{T}$ cells $(51,52)$, was markedly reduced on both $\mathrm{IgM}^{+}$and $\mathrm{IgM}^{-}$memory B cells of $c G A S^{-/-}$mice as compared with WT controls (Figure $6 \mathrm{E}$ ). We therefore concluded that ongoing infection is associated with poor GC function and memory B cell output in $c G A S^{-/-}$mice.

cGAS has no cell-intrinsic effects on $C D 4^{+} T$ cell or B cell GC development. Since we had observed differences in the formation of GCs in $c G A S^{-1-}$ mice as compared with WT controls, we tested whether cGAS affected $\mathrm{CD}^{+} \mathrm{T}$ cells or $\mathrm{B}$ cell differentiation in a cell-intrinsic fashion by constructing congenically marked mixed bone marrow chimeric mice. Six weeks after a 1:1 input of $2.5 \times 10^{6}$ bone marrow cells from either CD45.1 
A
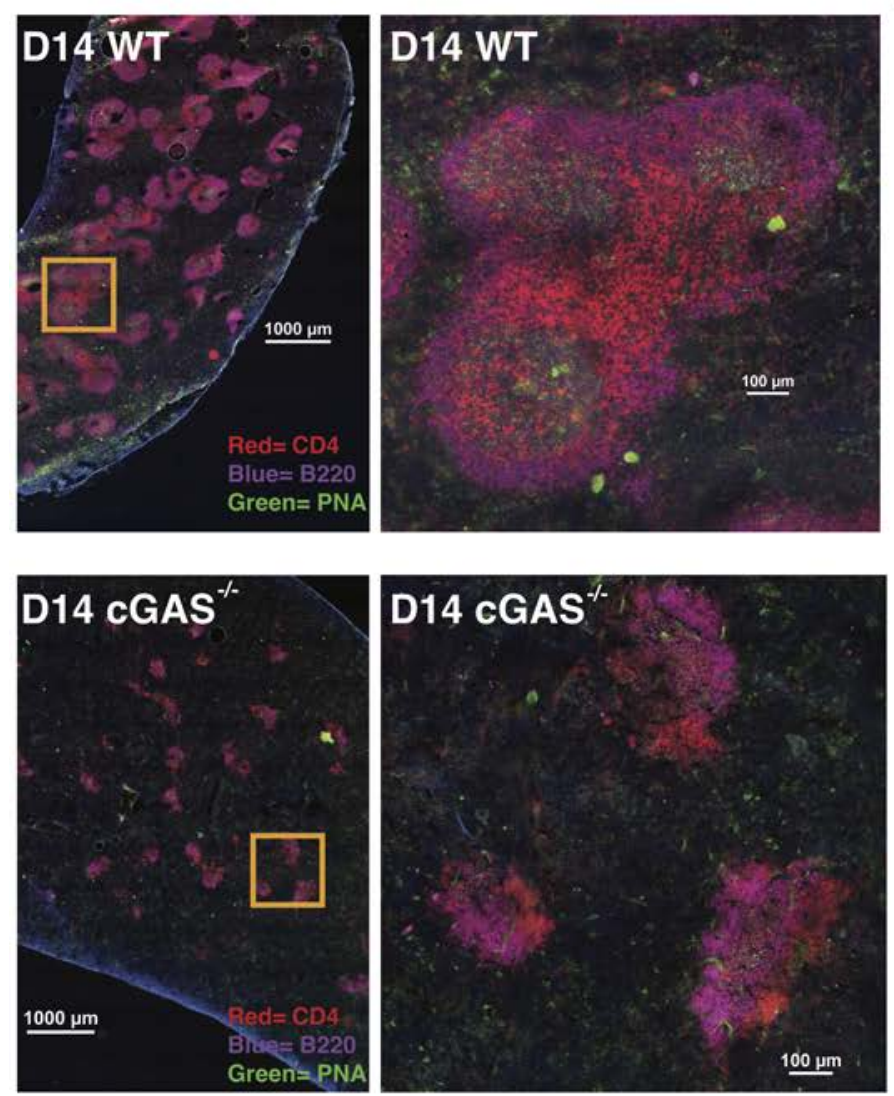

C

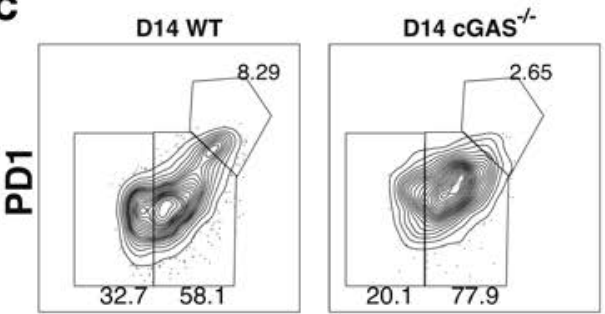

CXCR5

D

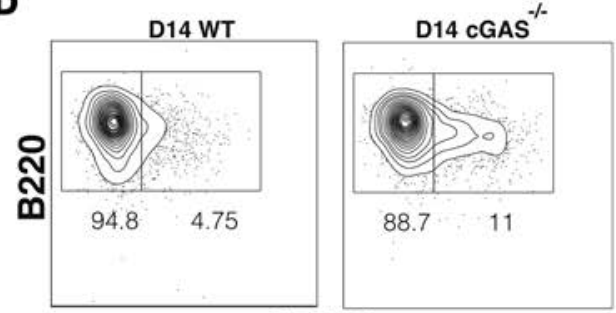

CD138
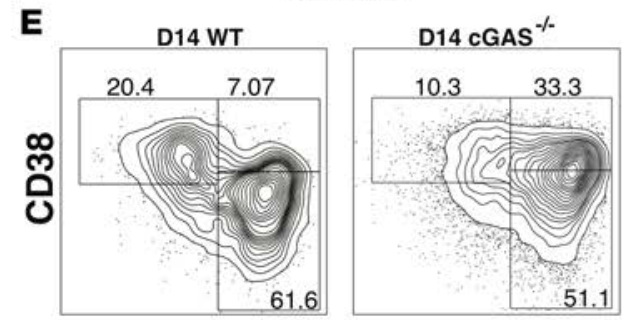

GL7
B

D14 MSP1-Specific lgG2c

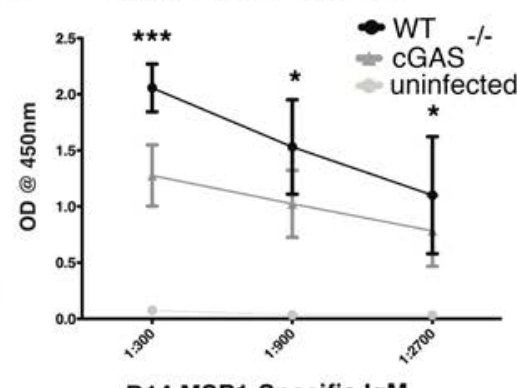

D14 MSP1-Specific IgM

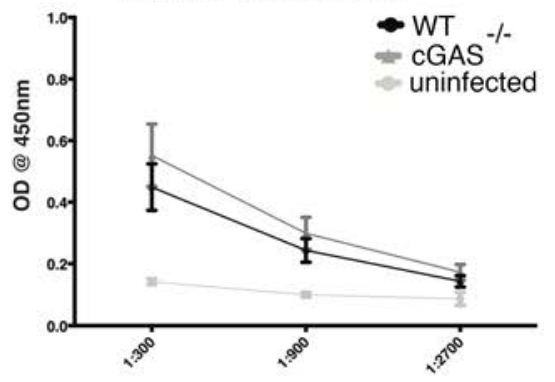

Figure 5. Absence of cGAS impairs germinal center formation and decreases production of class-switched antibody. (A) Sections of spleen harvested 14 days after infection stained with antibodies against PNA, B220, and CD4 (original magnification, $\times 20$ ). Follicles were counted, and the percentage of follicles with clear $\mathrm{PNA}^{+}$staining was determined. $77 \% \pm 10 \% \mathrm{PNA}^{+}$follicles were in WT mice as compared with $33 \% \pm 9 \%$ in $\mathrm{CCAS}^{-/}$ mice. $P=0.0052$ by Student's $t$ test. Sections representative of 3 biological replicates from 3 separate experiments are shown. Scale bar: 1,000 $\mu \mathrm{m}$ (left); $100 \mu \mathrm{m}$ (right). (B) Quantification of MSP1 IgG2c and IgM by ELISA in the serum 14 days after infection. Data represent 7 biological replicates pooled from 3 separate experiments. ${ }^{*} P<$ $0.05,{ }^{* *} P<0.01$. (C) Representative flow plots of enriched $C D 44^{+} \mathrm{CP} 66^{+}{ }^{+}$XXR5 $-\mathrm{T}$ effector cells (Teff), CXCR5 $5^{\text {int }}$ Tfh cells, and CXCR5 ${ }^{\text {hi }}$ CC Tfh cells. Data represent at least 9 biological replicates pooled from 3 separate experiments. (D) Representative flow cytometry plots used to identify $\mathrm{CD} 138^{+} \mathrm{M}$ $S P 1^{+} B$ cells. Data represent at least 7 biological replicates pooled from 3 separate experiments. $P$ $=0.0002$. (E) Representative flow plots demonstrating CD38+CL7- memory B cells, CD38 ${ }^{+} \mathrm{CL7} 7^{+} \mathrm{GC}$ precursors, and CD38-CL7+ $G C$ B cells. Data represent at least 7 biological replicates pooled from 3 separate experiments. Statistical analyses were performed using the unpaired Student's $t$ test. ${ }^{*} P$ $<0.05,{ }^{* *} P<0.01$. Error bars represent SD. 
mice (WT) or $c G A S^{-/-}$(CD45.2) mice, chimeric mice were infected with $P$. yoelii $17 X N L$ (Figure 7A). Fourteen days after infection, flow cytometric characterization was performed. No difference was observed in the ratio of congenically marked $\mathrm{CD}^{+} \mathrm{T}$ cells or $\mathrm{B}$ cells when compared with the ratio of congenically marked cells tested prior to infection (data not shown). No differences were observed in the percentage of $\mathrm{GP}^{+} 6^{+}$-specific $\mathrm{CD}^{+}$GC T cells (Figure 7B). No differences were observed in MSP1-specific B cells (Figure 7C). Thus, we concluded cGAS deficiency has no cell-intrinsic effects at time points where we had previously observed differences in the adaptive response in $c G A S^{-1-}$ mice as compared with controls.

Clearance of parasite by atovaquone restores $B$ cell memory and GC responses. We have previously observed profound effects of blood-stage infection on the development of liver-stage-specific germinal center and memory formation (38). Plasmodium infection has been associated with ongoing splenic disruption in mice, monkeys, and humans (52-56). We hypothesized that the reduced GC function we observed in $c G A S^{\prime-}$ mice could therefore be due to indirect effects from exacerbated infection. To test this possibility, we admin istered the drug atovaquone (MilliporeSigma) (which kills blood-stage parasites) to $c G A S^{-1-}$ and control mice 7 days after infection. We chose this regimen because we had observed differences in parasitemia in WT and $c G A S^{-1}$ mice starting after day 7, and other antimalarial agents have known immunomodulatory properties $(57,58)$. Parasites were cleared from the blood within 2 days of starting atovaquone treatment (data not shown). We assessed the antigen-specific $\mathrm{CD}^{+} \mathrm{T}$ cells and $\mathrm{B}$ cells from experimental or control mice on day 22 after infection when we had previously seen differences in GC responses. Drug clearance of the parasite resulted in the complete restoration of both the number and percentage of GC Tfh cells (Figure $8 \mathrm{~A})$. Drug treatment reduced the percentage of $\mathrm{CD} 138^{+} \mathrm{MSP} 1^{+}$plasmablasts to levels similar to WT mice (Figure $8 \mathrm{~B}$ ). Coincident with the reduction in plasmablasts, treatment with atovaquone restored the number and percentage of GC B cells in $c G A S^{-1-}$ mice to levels similar to WT mice (Figure $8 \mathrm{C}$ ). We therefore concluded that both the compromise in GC function and increased rate of plasmablast formation we had observed in $c G A S^{-1-}$ mice were dependent upon ongoing infection and could be restored by clearing the infection with atovaquone. These data demonstrate that the effects of $c G A S$ deletion on the GC response are indirect and reflect an important role for cGAS in reducing parasitemia but cGAS is dispensable for development of the GC response.

\section{Discussion}

Our investigations reveal that cGAS contributes to parasite control, which is essential for the formation of GC-derived humoral immunity. The ability to simultaneously identify and analyze endogenous polyclonal antigen-specific $\mathrm{CD}^{+} \mathrm{T}$ cells and B cells responding to Plasmodium has revealed a profound effect of innate immune control of parasitemia. Control of parasitemia is important for timely development of the GC response, a response critical for both the efficient generation of high-affinity antibodies and durable immune memory (59-62). Furthermore, our ability to track parasitemia throughout the course of infection combined with the potential to rapidly clear blood-stage infection with atovaquone provided us with an elegant system in which the effects of innate control of parasite burden could be disentangled from the effects of early innate signals on the adaptive immune response. Specifically, the collapse of the GC response in $c G A S^{-1-}$ mice as parasitemia persisted could be abolished with clearance of the parasite via drug treatment, emphasizing the destructive effects blood-stage Plasmodium infection can have on the host GC. From a translational standpoint, our findings suggest that ongoing blood-stage malaria infection is generally deleterious to the GC response, providing insight into how improved immunity may develop under chemoprophylaxis against blood-stage infection (63). Previously, it had been demonstrated that blood-stage infection compromised the development of liver-stage antibodies $(38,64)$. The concept that an ongoing blood-stage infection also reduces the quality of a blood-stage humoral response is, however, to our knowledge novel.

Importantly, our findings demonstrate that cGAS contributes to parasite control in response to a nonlethal P. yoelii infection. Our findings emphasize the importance of balancing immune-mediated protection and pathology during the primary immune response. In contrast to recent reports documenting increased survival in $c G A S^{-/-}$mice during infection with the closely related lethal strain P. Yoelii CM(6), we determined that absence of cGAS was associated with poor parasite control late during infection with the nonlethal $P$. yoelii $17 X N L$. We confirmed that $c G A S^{-/}$mice had worsened outcomes with the lethal strain P. yoelii $17 X L$. While differences in type I IFN production at the protein level were present late during infection, cGAS may drive production of other cytokines. For example, increased serum levels of IL-12, TNF, and CCL2 have been reported in mice treated with exogenous cGAMP (the secondary messenger produced by cGAS) $(65,66)$. 
A

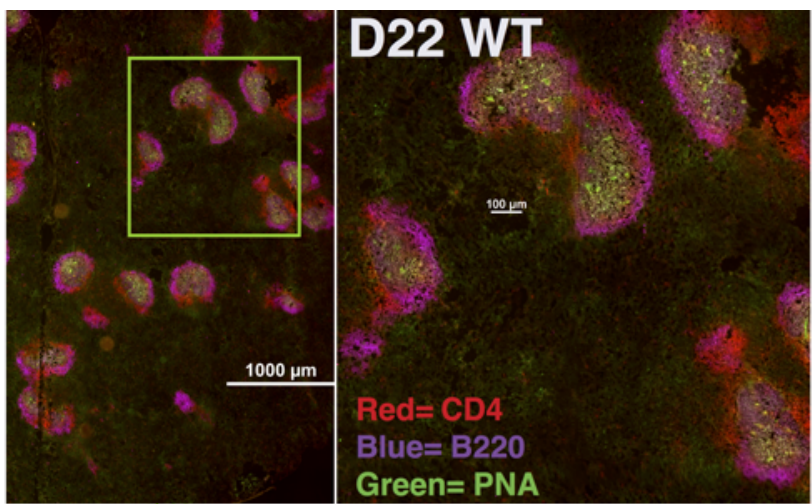

Green $=$ PNA
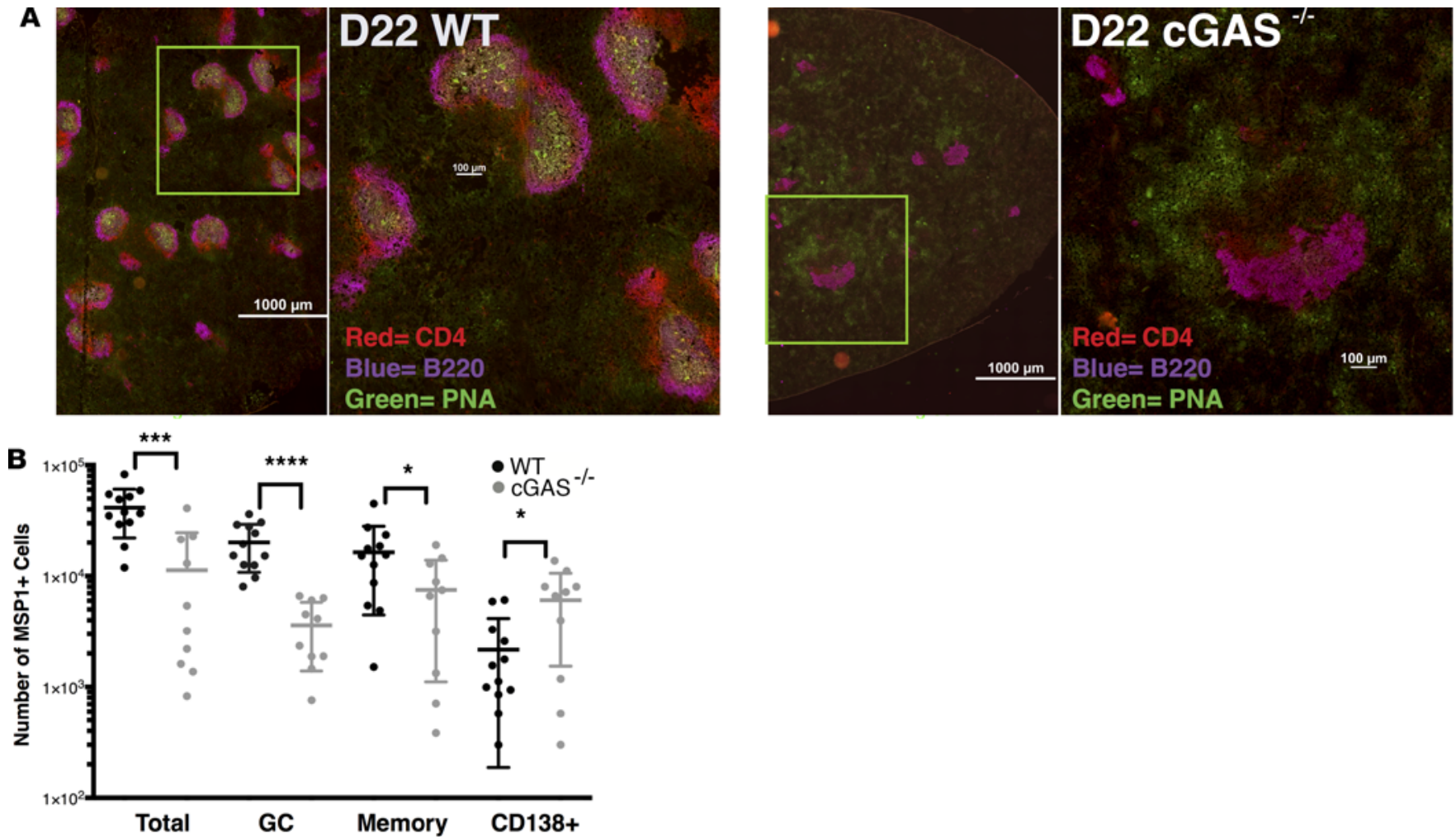

C

D22 MSP1-Specific IgG2c

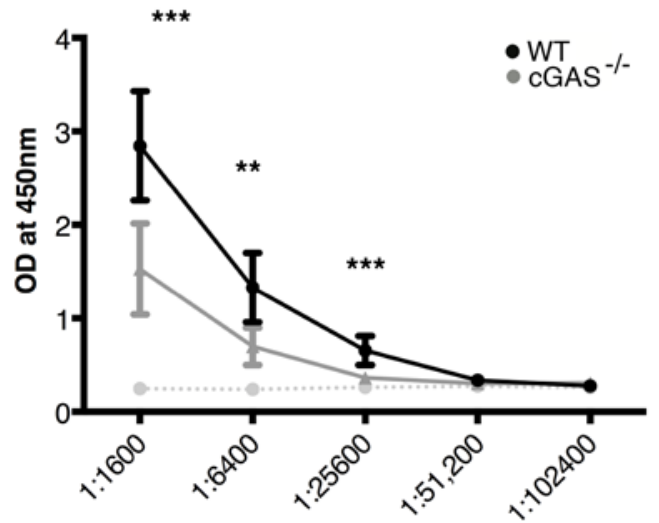

Dilution

E

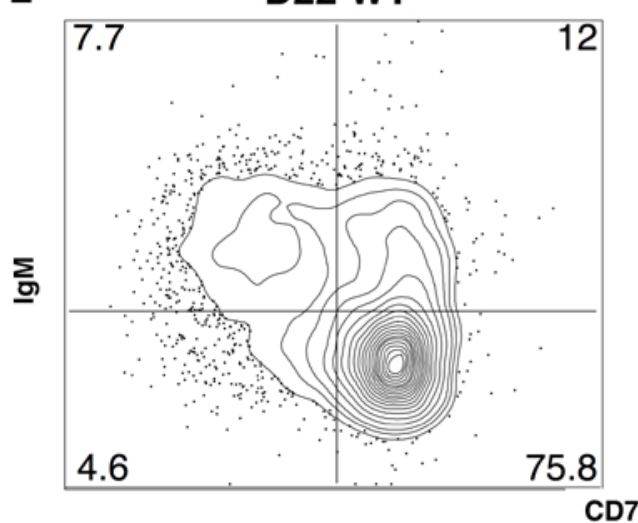

D22 MSP1-Specific IgM

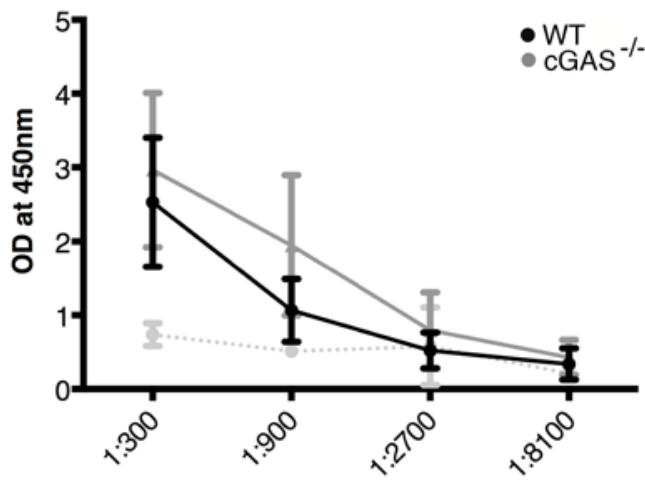

Dilution

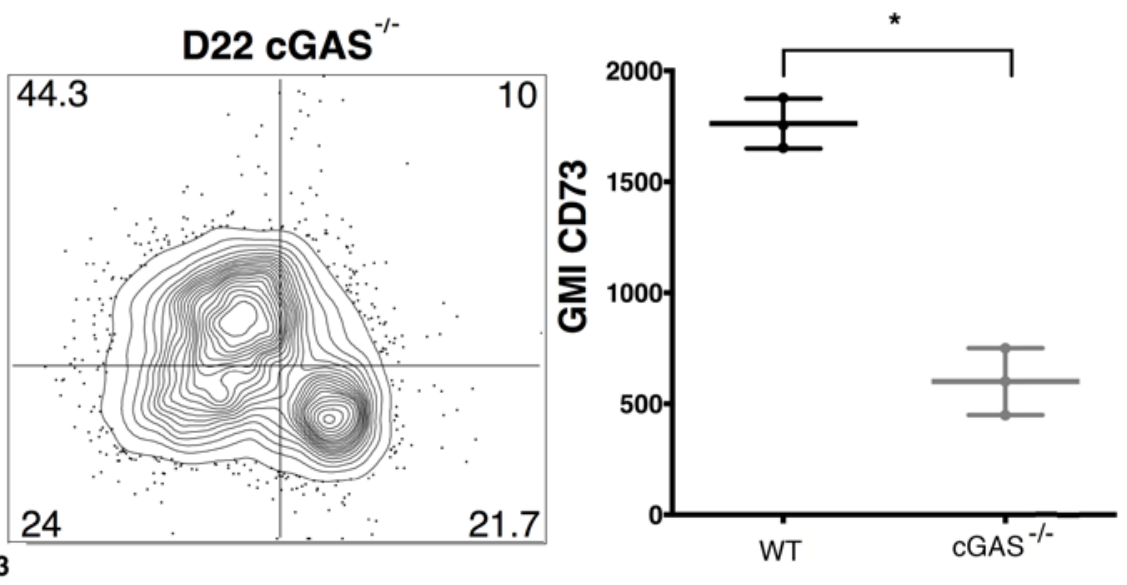


Figure 6. Prolonged reduction in germinal center response is associated with reduced memory B cell formation in cGAS $^{-/-}$mice. (A) Spleen sections were stained with antibodies against PNA, B220, and CD4. Scale bar: 1,000 $\mu \mathrm{m}$ (first and third images); $100 \mu \mathrm{m}$ (second and fourth images). (B) Quantification of $\mathrm{MSP}^{+} \mathrm{B}$ cells subsets 22 days after infection with Plasmodium yoelii $17 \mathrm{XNL}$. Error bars represent SD. ${ }^{*} P>0.05,{ }^{* *} P<0.001,{ }^{* * * *} P<0.0001$, by unpaired Student's $t$ test. (C and $\mathbf{D})$ Quantification of serum levels of MSP1-specific IgC2c and IgM 22 days after infection. Data represent 7 biological replicates from 3 pooled experiments. Statistical analysis was performed using the unpaired Student's $t$ test. Error bars represent SD. ${ }^{* *} P<0.01,{ }^{* * *} P<$ 0.001. (E) Mean fluorescent intensity of CD73 on MSP1-specific memory B cells that are CD38 $/$ GL7- 22 days after infection. Representative data are shown representative of 2 separate experiments. The geometric mean intensity (GMI) of $c$ CAS ${ }^{-1-}$ mice was $600 \pm 151$ as compared with versus $1,763 \pm 112$ in WT controls. $P<0.001$ by unpaired Student's $t$ test. Error bars represent SD. ${ }^{*} P<0.05$.

This finding mirrors the potentially dichotomous role of type I IFN in lethal models as compared with nonlethal models of blood-stage malaria. In murine models of Plasmodium in which death is associated with immunopathology, such as $P$. berghei, the absence of IFNAR leads to reduced tissue pathology and reduced mortality $(24,67-69)$. Treatment with IFN- $\alpha$ or IFN- $\beta$, both prior to and during infection, however, reduces lethality, implying a role for early parasite control in contributing to lethality $(70,71)$. In nonlethal murine models, the role of IFNAR signals vary depending on the strain of parasite and experimental conditions. During P. chabaudi infection, $I F N A R^{-/-}$mice had elevated (72), unchanged (21), or reduced parasitemia $(73,74)$. The role of type I IFN in the control of $P$. yoelii (another model of nonlethal blood-stage malaria) is also variable, as disruption of type I IFN signals has been demonstrated to either increase (75) or decrease parasite burden $(28,33)$. Furthermore, increased type I IFN signals associated with knockout of the IRF3 repressor FOSL1 lead to reduced parasite burden (76).

Although our results differ from the reports of Zander et al. with respect to the effects of type I IFN signals on parasite control during P. yoelii 17XNL infection (33), the discrepancy may be due to the timing of the depletion of type I IFN, as they administered antibody on days $-1,2$, and 4 . Similar to others (75), we observed that type I IFN signals persist beyond day 4 after infection with $P$. yoelii and may be important for innate effector mechanisms. Sebina et al. also recently reported reduced parasitemia in $I F N A R^{-/-}$mice as compared with control mice infected with P. yoelii $17 X N L$ starting at day 14 after infection (28). Another potential explanation for different experimental outcomes could be either mouse strain differences or differences in the microbiome, both of which have substantial effects on the host response to infection in murine models of blood-stage malaria (77-79). We used littermate controls to minimize the contribution of microbiome or strain background to our observations. Differences in parasite virulence may also account for some of the observed differences. We avoid using parasites passaged more than 3 times without recirculation through a mosquito, as repeated passage of murine strains of malaria has been demonstrated to increase virulence and substantially alter the host response (80).

Our observations that clearance of blood-stage malaria with atovaquone eliminates differences in the T cell-dependent GC response should prompt future studies to consider drug treatment as a control in models with divergent parasite burden. When infection is ongoing, there was impairment of both malaria-specific GC Tfh CD4 ${ }^{+}$T cells and GC B cells that could be restored with pharmacologic clearance of the parasite. Although antibodies are important for clearance of blood-stage malaria (81-84), whether manipulation of innate cytokines or PRRs directly affects the humoral response to blood-stage malaria infection should be determined after normalization of the parasite burden.

We propose that one mechanism for the poor GC response observed in the $c G A S^{-1-}$ mice is due to increased formation of plasmablasts. This occurs in a cGAS-independent manner late after initial priming and expansion and occurs in conjunction with a reduction in the number of GC B cells. Since plasmablasts are thought to be short lived and not contribute to the memory pool (85), our data support epidemiologic observations that high levels of ongoing parasite transmission are associated with poor-quality, short-lived B cell responses (86-88). In contrast to the reduced number of MSP1-specific B cells, we determined that ongoing infection had no effects on the total number of endogenous antigen-specific $\mathrm{CD} 4^{+} \mathrm{T}$ cells but did affect the formation of GC Tfh CD4 ${ }^{+} \mathrm{T}$ cells. We have previously demonstrated that blood-stage infection disrupts the GC response to liver-stage antigens and that blood-stage infection is associated with alterations in chemokine expression, including CXCL10, that can be reversed by treatment with atovaquone (38). It is likely that the elevated parasite burden in cGAS mice leads to persistent alterations in chemokine signals. Differences in antigen burden may also affect the poor GC formation in the $c G A S^{/-}$mice. Using model antigen systems (NP-CPG), it has recently been demonstrated that excess antigen diverts GC precursors toward a plasmablast fate (89). 
A
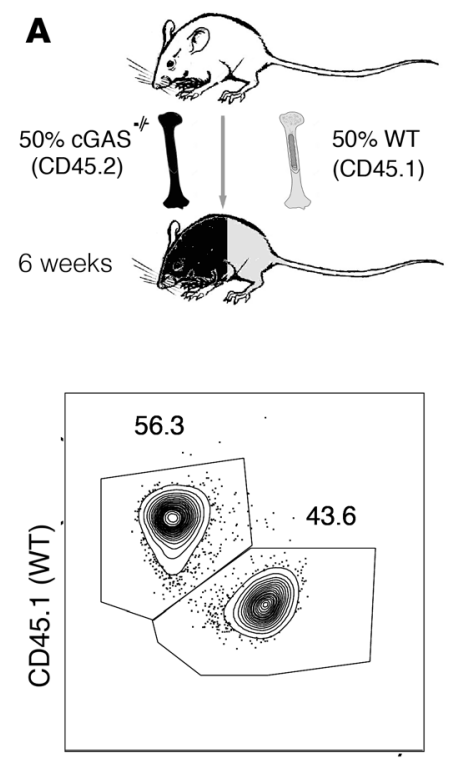

CD45.2 (cGAS ${ }^{-}$)

C

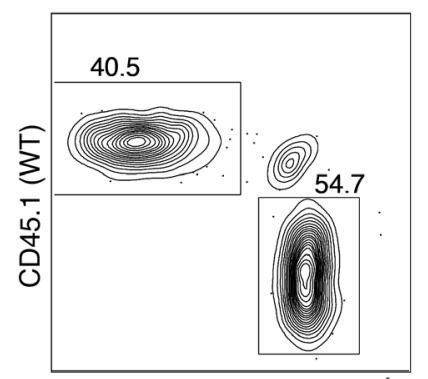

CD45.2 ( (GAS $\left.^{-1}\right)$

B
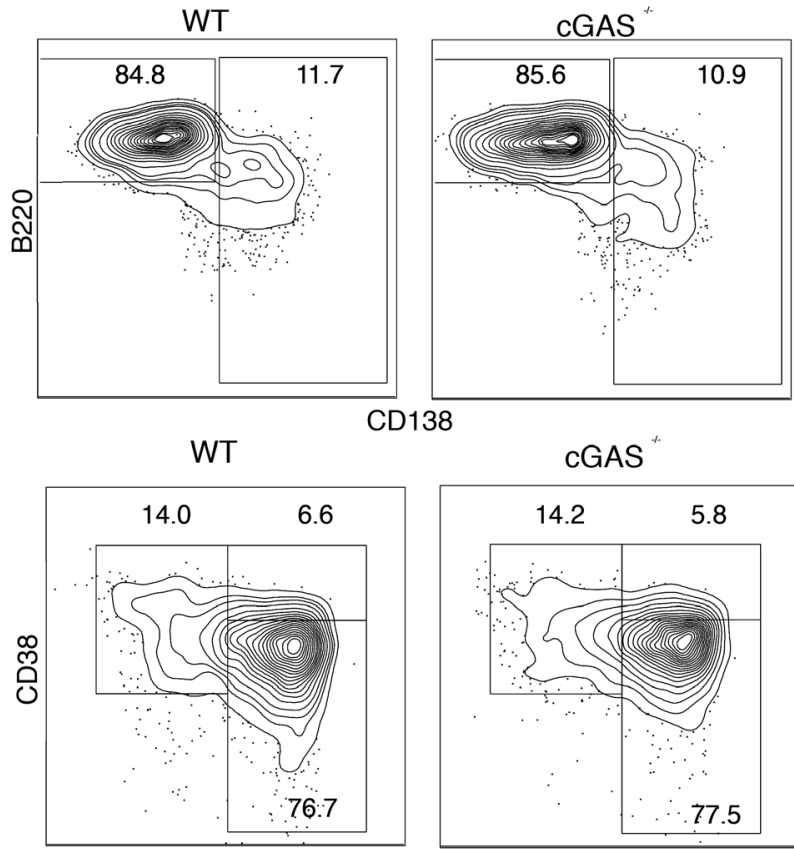

GL7

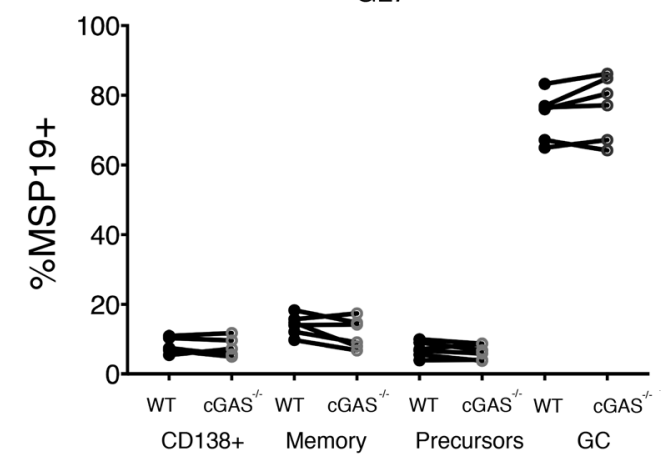

WT
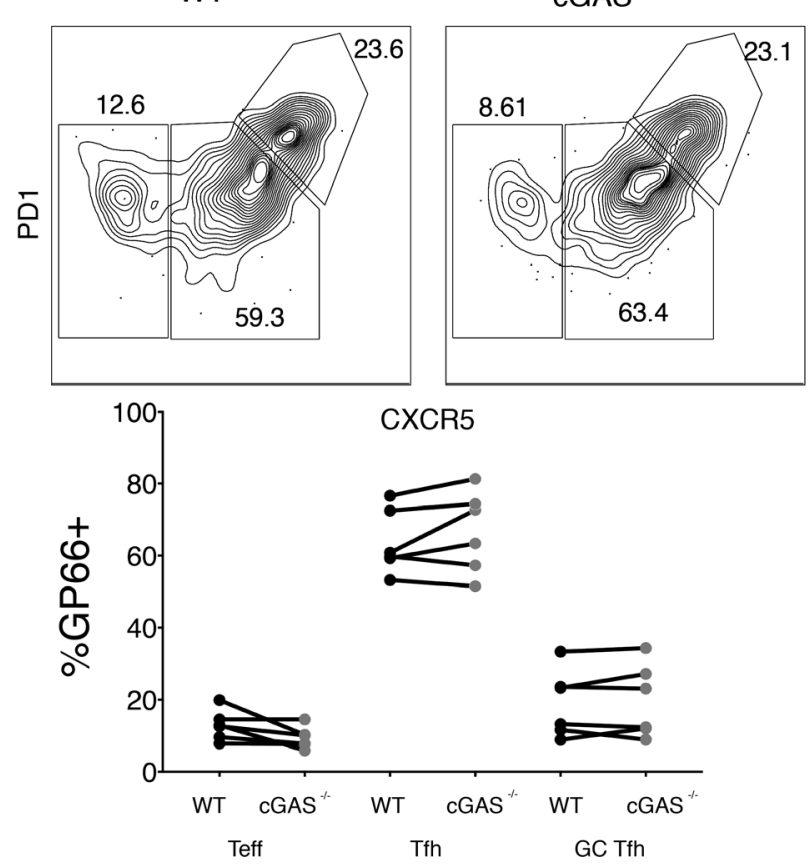

Figure 7. cGAS has no cell-intrinsic activity in antigen-specific $\mathrm{CD4}^{+} \mathrm{T}$ cells or $\mathrm{B}$ cells.

(A) Representative flow plot demonstrating CD45.1 and CD45.2 chimerism among total MSP1-specific B cells 14 days after infection. (B) CD138+ plasmablast frequency (top row) and $\mathrm{CL7} 7^{+}$precursor and GC B cells (bottom row) are similar in WT compared to cGAS $^{-1-} \mathrm{MSP}^{+} \mathrm{B}$ cells. Plots are representative of 6 mice from 2 separate experiments. Statistical analysis was performed by the paired Student's $t$ test. (C) Representative flow plot demonstrating CD45.1 and CD45.2 chimerism among total $\mathrm{CP}_{66}{ }^{+} \mathrm{CD} 44^{+} \mathrm{CD} 4^{+} \mathrm{T}$ cells 14 days after infection. Frequencies of CXCR5 ${ }^{-}$Teff, CXCR5 int $T$ Th, and CXCR5 ${ }^{\text {hi }}$ GC Tfh cells are similar between WT and $\mathrm{CGAS}^{-1-}$ populations. Plots are representative of 6 mice from 2 separate experiments. Statistical analysis was performed by the paired Student's $t$ test. 
JCl insIGHT

RESEARCH ARTICLE

A D22 WT

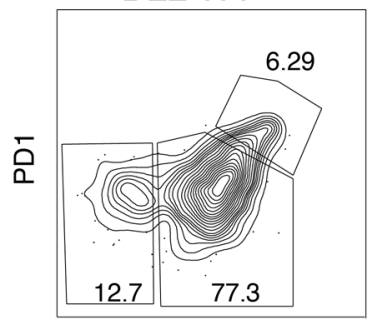

$\mathrm{D} 22 \mathrm{cGAS}^{-/-}$

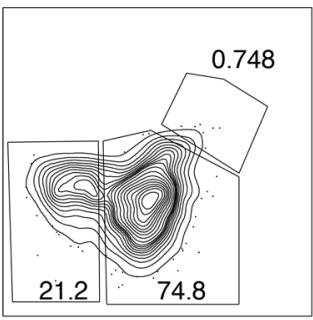

CXCR5
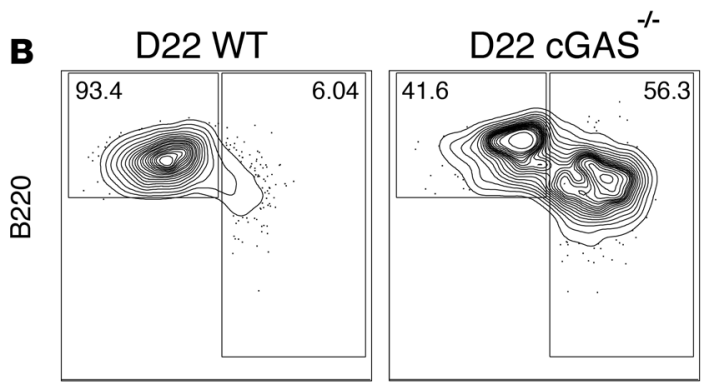

CD138
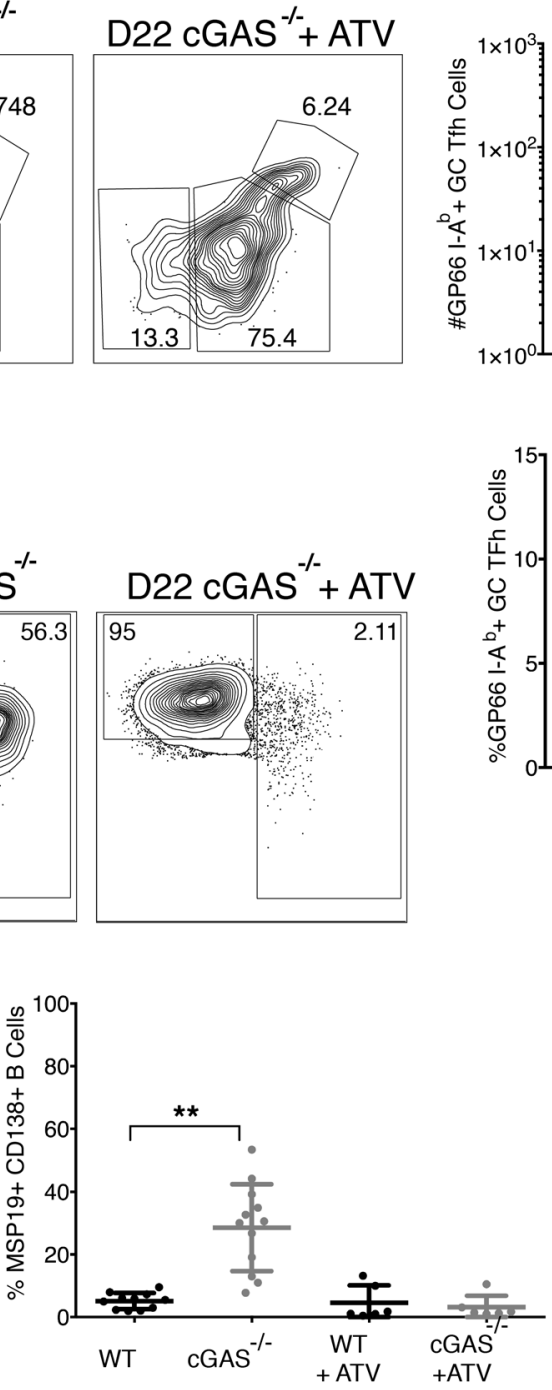
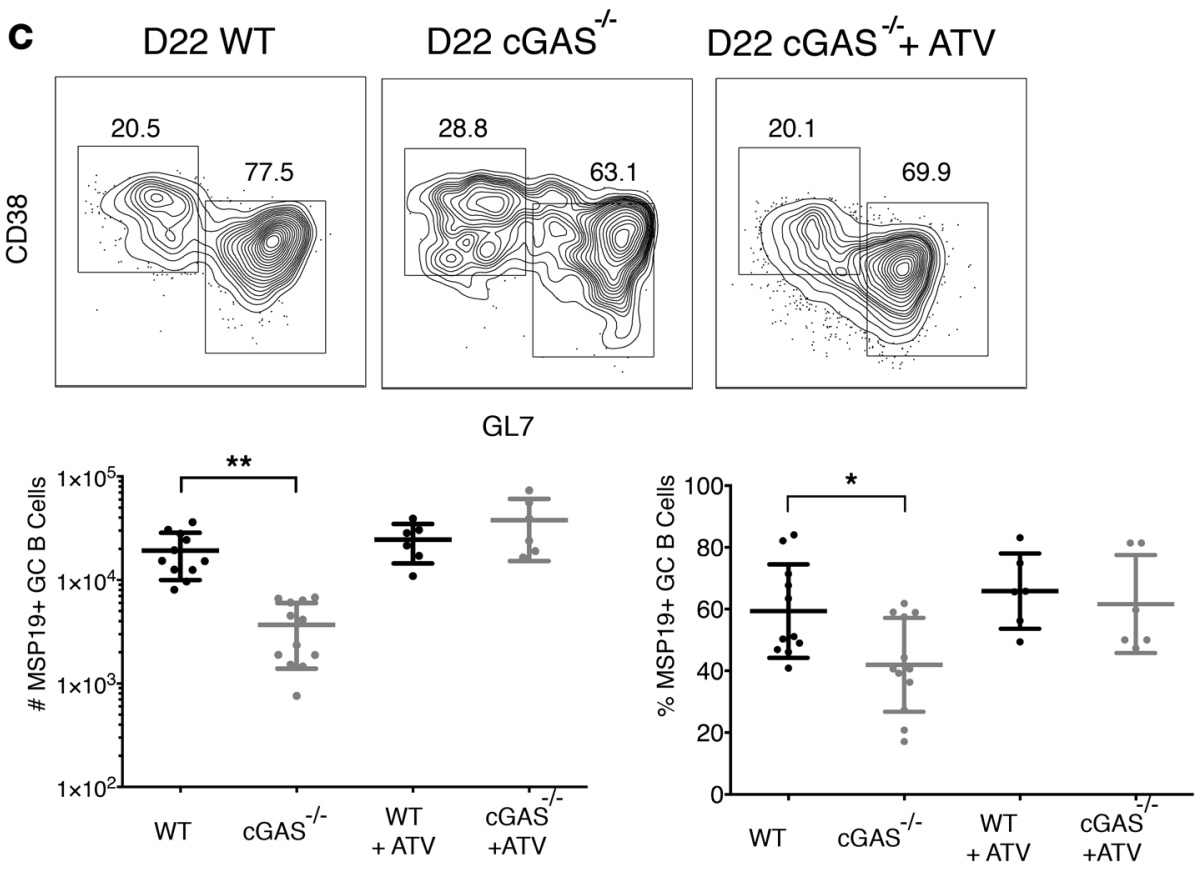

insight.jci.org https://doi.org/10.1172/jci.insight.94142

13 
Figure 8. Clearance of parasite with atovaquone restores germinal center CD4+ $\mathbf{T}$ cells and B cell responses. (A) WT and $c C A S^{-1-}$ mice were infected with $10^{6}$ Plasmodium yoelii 17XNL-infected erythrocytes via the intraperitoneal route. Starting at day 7, mice were treated with atovaquone for 5 days via daily intraperitoneal injection. GP66+CD4+ $T$ cells enriched from spleen and lymph nodes were examined for GC Tfh differentiation using antibodies against CXCR5 and PD1. Because data were nonparametric with different SDs, statistical analysis for both number and percentage was performed using a Kruskal-Wallis test with Dunn's post-hoc comparison. WT mice were selected as the comparator group. ${ }^{*} P<0.05,{ }^{* * *} P<0.001$. (B) MSP1+ B cells were identified and CD138 plasmablasts were quantified. Statistical analysis was performed using Kruskal-Wallis test with Dunn's post-hoc comparison. WT mice were selected as the comparator group. In $\mathrm{CGAS}^{-1-}$ mice treated with atovaquone, there were CD138 $\mathrm{MSP}^{+} 3.169 \% \pm 3.6 \%(P=0.92) .{ }^{*} P<0.05$, ${ }^{* * *} P<0.001$. (C) MSP1+ $\mathrm{CC} \mathrm{B}$ cells were quantified. ${ }^{*} P<0.05$, ${ }^{* *} P<0.001$. Statistical analysis was performed using Kruskal-Wallis test with Dunn's post-hoc comparison. WT mice were selected as the comparator group. Data represent at least 6 biological replicates from 2 separate experiments. Error bars represent SD.

We determined that there was no difference in $\mathrm{CD} 4^{+} \mathrm{T}$ or $\mathrm{B}$ cell development when the host environment was normalized using mixed bone marrow chimeric mice, demonstrating that cGAS does not act in a cell-intrinsic manner. Furthermore, elimination of the parasite with antimalarial drugs, restored late GC $\mathrm{Tfh} \mathrm{CD}^{+} \mathrm{T}$ cell function in $c G A S^{-1-}$ mice, demonstrating that the deficiencies in $\mathrm{CD}^{+} \mathrm{GC}$ Tfh formation we had observed were due to elevations in the parasite burden rather than an intrinsic programming defect.

The finding that ongoing blood-stage infection impairs the development of GC-derived humoral immunity to blood-stage antigens has clinical implications. For example, children in areas of high parasite transmission had worse vaccine response rates to the RTS'S vaccine than children in lower transmission areas, with no evidence of boosting of CSP-specific antibodies with natural infection or vaccination (90). A vaccine capable of reducing the magnitude or duration of blood-stage malaria could reasonably be anticipated to improve the quality of humoral response against Plasmodium and potentially other pathogens. Areas where malaria is eliminated consistently experience a decrease in mortality greater than can be attributed to malaria alone, typically associated with decreases in unrelated infections (91, 92). The role that persistent cGAS-mediated innate signaling plays in the control of parasitemia should remain an active area of exploration. Therefore, despite minimal effects on the adaptive system during acute priming of the response to blood-stage malaria, the cGAS-STING axis remains a target for manipulation for improving clinical outcomes in blood-stage malaria.

\section{Methods}

Mice. Male 6-to 8-week old C57BL/6J mice were purchased from Jackson ImmunoResearch Laboratories and were maintained and bred under specific pathogen-free conditions, per the institutional guidelines at the University of Washington. IFNAR ${ }^{-/-}$, Tmem $173^{g t / g t}\left(S T I N G^{-/-}\right)$, and $c G A S^{-/-}$mice were provided by the MG and Dan Stetson (University of Washington). IFNAR ${ }^{-/-}$and $c G A S^{-/-}$mice were bred as heterozygotes for experiments with littermate controls after genotyping as previously described $(93,94)$. All mice used for experimental purposes were male and aged 6-10 weeks.

Mixed bone marrow chimeras. Mixed bone marrow chimeras were generated as described previously (49). Briefly, bone marrow cells were depleted of $\mathrm{T}$ and NK cells, and congenically marked WT (CD45.1) cells and $\mathrm{cGAS}^{-/}$(CD45.2) cells were mixed in equal portions. Recipient mice (CD45.1/CD45.2) were lethally irradiated (10 Gy) and injected with total bone marrow cells and given enrofloxacin-treated water for 6 weeks.

Parasites. The 17XNL strain of $P$. yoelii was modified at a dispensable genetic locus ("p230p") to stably incorporate an expression cassette for the $P$. yoelii hep 17 gene tagged at the C-terminus with amino acids 51-84 of the GP from LCMV. Standard methods were used to create and integrate a linearized pDEF construct as well as to select transgenic parasites and to obtain two pure, clonal populations for further study. Confirmation of the presence of the transgenic locus was determined by genotyping PCR. P. yoelii 17XNL expressing the $\mathrm{GP}_{66}$ epitope were maintained as frozen blood stocks and passaged through donor mice with no more than 3 passages prior to recirculation through mosquito inoculation. Infections were induced by intraperitoneal injection of $10^{6}$ parasitized red blood cells from the blood of donor mice at $1 \%-5 \%$ parasitized red blood cells.

Tetramer production. Biotinylated I-A ${ }^{\mathrm{b}}$ LCMV GP 66-77 DIYKGVYQFKSV monomers were obtained from the NIH tetramer core and tetramerized with SA-APC as previously described (95). For antigen-specific B cell experiments, a 14-kDA truncated carboxy terminus of $P y \mathrm{MSP} 1$ was cloned, purified, biotinylated, and tetramerized with streptavidin-PE (Prozyme) as previously described $(38,96)$. Decoy reagent to detect B cells specific for tetramer components was constructed as previously described $(17,43)$.

Cell enrichment, flow cytometry, and antibodies. Single-cell suspensions of spleen and cervical, mediastinal, axillary, brachial, pancreatic, renal, mesenteric, inguinal, and lumbar lymph nodes (SLO) were prepared by mashing through Nitex mesh (Sefar, B0015H309U) and resuspending in 2\% FBS and Fc block (2.4G2). Hemozoin was 
depleted via running cell suspension over magnetized LS columns (Miltenyi Biotec), and the resulting fraction was stained with $\mathrm{GP}_{66} \mathrm{I}: \mathrm{A}^{\mathrm{b}}$-APC tetramer and enriched by magnetic beads as previously described (95). The unbound fraction was then stained with decoy $\gamma$ reagent at a concentration of $10 \mathrm{nM}$ at room temperature for 15 minutes followed by MSP1 tetramer for 30 minutes on ice, washed, and then stained with anti-PE beads prior to a repeated magnetic enrichment. All bound cells then were stained with antibodies shown in Supplemental Table 1, detected on an LSRII Flow Cytometer (BD Biosciences), and analyzed using Flowjo 9.94 (Treestar).

ELISA. 96-well ELISPOT plates (Millipore) were coated overnight at $4^{\circ} \mathrm{C}$ with $\mathrm{MSP} 1^{+}$protein (BEI resources) at $1 \mu \mathrm{G} / \mathrm{ml}$. Plates were then blocked with $5 \%$ dehydrated milk prior to sample incubation. Plates were incubated with serially diluted serum. Bound antibodies were detected using either IgM biotin (clone II/41) (Southern Biotech) or IgG2c biotin (clone 5.7) (Southern Biotech), followed by streptavidin-HRP (BD). Absorbance was measured at $450 \mathrm{~nm}$ using an iMark Microplate Reader (Bio-Rad). IFN- $\gamma$ concentration was calculated using the Ready-SET-Go! ELISA kit (eBioscience) as per the manufacturer's instructions.

Histology. Mouse spleens were flash frozen in liquid nitrogen prior to cutting on cryotome. 10-micron sections were cut and fixed for 10 minutes with ice-cold acetone. After washing, sections were blocked with 20\% FBS for at least 2 hours. Then, sections were stained with CD4-bio (RM4.4), followed by SA-AF568, B220-AF647, and PNA-FITC. Sections were imaged using the Eclipse 90i Advanced Automated Research Microscope (Nikon). Scale bars were placed using Nikon software.

Quantitative PCR. Mouse spleens were stored at $-80^{\circ} \mathrm{C}$ in RNAlater (Qiagen) prior to homogenization using a bead homogenizer and extraction with the RNeasy Mini kit (Qiagen). cDNA was then generated using a Superscript Vilo cDNA synthesis kit (Thermo Fisher). Quantitative PCR was then performed using PowerSYBR 2x master mix (Thermo Fisher) and primers indicated in Supplemental Table 2 on a CFX 1000 Thermal Cycler (Bio-Rad). All Ct values were normalized to HPRT, and quantitation was calculated using the delta-delta CT method.

Type I IFN bioassays of mouse cells. Luciferase activity was measured in L929 cells stably expressing an ISRE-luciferase reporter as previously described (25). $5 \times 10^{4}$ L929-ISRE cells were plated at a ratio of 10 splenocytes to 1 reporter cell in a 96-well plate. Cells were lysed and luciferase activity was measured using the Luciferase Assay System (Promega) and Centro LB 960 Microplate Luminometer (Berthold Technologies).

Statistics. When data were parametric, unpaired, 2-tailed Student's $t$ tests were applied to determine the statistical significance of the difference between groups with Prism 6 software (GraphPad). For analysis of mRNA, a 2-tailed Mann-Whitney $U$ test was utilized, as the data were nonparametric. For analysis of multiple groups with different SDs and nonparametric data, the Kruskal-Wallis test was used with the Dunn post-hoc test for multiple comparisons.

Study approval. All experiments involving animals were performed in accordance with the guidelines of and with the approval of the University of Washington Institutional Animal Care and Use Committee.

\section{Author contributions}

WOH, MP, and WCL designed experiments and analyzed data. WOH and HMK performed experiments. DNS provided PyMSP1 protein. NSB, SHIK, and SEL designed and constructed $P y G P 66^{+}$parasites. JAH and MG provided expertise and critically reviewed the manuscript. MG provided IFNAR and STING signaling mutant $\left(\right.$ Tmem $\left.^{g t / g t}\right)$ mice. WOH, WCL, and MP wrote the manuscript.

\section{Acknowledgments}

We thank Brian Hondowicz for experimental advice and technical assistance. This work was supported by grants to WOH (NIH grant T32 AI007044-39), MG (NIH grants R01 AI04002 and U19 AI083019), and MP (NIH grant R01 AI118803, Burroughs Wellcome Fund 1016766).

Address correspondence to: Marion Pepper, University of Washington, Department of Immunology, Campus Box 358059, 750 Republican Street, Seattle, Washington 98109, USA. Phone: 206.221.5512; Email: mpepper@uw.edu.

SEL's present address is: Department of Biochemistry and Molecular Biology, Center for Malaria Research, Pennsylvania State University, University Park, Pennsylvania, USA. 
1. GBD 2013 Risk Factors Collaborators, et al. Global, regional, and national comparative risk assessment of 79 behavioural, environmental and occupational, and metabolic risks or clusters of risks in 188 countries, 1990-2013: a systematic analysis for the Global Burden of Disease Study 2013. Lancet. 2015;386(10010):2287-2323.

2. Hansen DS, Obeng-Adjei N, Ly A, Ioannidis LJ, Crompton PD. Emerging concepts in T follicular helper cell responses to malaria. Int J Parasitol. 2017;47(2-3):105-110.

3. Gazzinelli RT, Kalantari P, Fitzgerald KA, Golenbock DT. Innate sensing of malaria parasites. Nat Rev Immunol. 2014;14(11):744-757.

4. Inoue S, Niikura M, Mineo S, Kobayashi F. Roles of IFN- $\gamma$ and $\gamma \delta$ T cells in protective immunity against blood-stage malaria. Front Immunol. 2013;4:258

5. Wykes MN, Good MF. What really happens to dendritic cells during malaria? Nat Rev Microbiol. 2008;6(11):864-870.

6. Yu X, et al. Cross-regulation of two type I interferon signaling pathways in plasmacytoid dendritic cells controls anti-malaria immunity and host mortality. Immunity. 2016;45(5):1093-1107.

7. Sun L, Wu J, Du F, Chen X, Chen ZJ. Cyclic GMP-AMP synthase is a cytosolic DNA sensor that activates the type I interferon pathway. Science. 2013;339(6121):786-791

8. Zhang X, et al. The cytosolic DNA sensor cGAS forms an oligomeric complex with DNA and undergoes switch-like conformational changes in the activation loop. Cell Rep. 2014;6(3):421-430.

9. Li XD, Wu J, Gao D, Wang H, Sun L, Chen ZJ. Pivotal roles of cGAS-cGAMP signaling in antiviral defense and immune adjuvant effects. Science. 2013;341(6152):1390-1394.

10. Zhang Y, et al. The DNA sensor, cyclic GMP-AMP synthase, is essential for induction of IFN- $\beta$ during Chlamydia trachomatis infection. J Immunol. 2014;193(5):2394-2404.

11. Andrade WA, et al. Type I interferon induction by Neisseria gonorrhoeae: Dual requirement of cyclic GMP-AMP synthase and Toll-like receptor 4. Cell Rep. 2016;15(11):2438-2448.

12. Collins AC, et al. Cyclic GMP-AMP synthase is an innate immune DNA sensor for Mycobacterium tuberculosis. Cell Host Microbe. 2015;17(6):820-828.

13. Lio CW, et al. cGAS-STING signaling regulates initial innate control of Cytomegalovirus infection. J Virol. 2016;90(17):7789-7797.

14. Diner EJ, et al. The innate immune DNA sensor cGAS produces a noncanonical cyclic dinucleotide that activates human STING. Cell Rep. 2013;3(5):1355-1361.

15. Zeng M, et al. MAVS, cGAS, and endogenous retroviruses in T-independent B cell responses. Science. 2014;346(6216):1486-1492.

16. MacLeod MK, McKee A, Crawford F, White J, Kappler J, Marrack P. CD4 memory T cells divide poorly in response to antigen because of their cytokine profile. Proc Natl Acad Sci USA. 2008;105(38):14521-14526.

17. Taylor JJ, et al. Deletion and anergy of polyclonal B cells specific for ubiquitous membrane-bound self-antigen. $J$ Exp Med. 2012;209(11):2065-2077.

18. Lelliott PM, McMorran BJ, Foote SJ, Burgio G. In vivo assessment of rodent Plasmodium parasitemia and merozoite invasion by flow cytometry. J Vis Exp. 2015;(98):e52736.

19. Malleret B, et al. Plasmodium vivax: restricted tropism and rapid remodeling of CD71-positive reticulocytes. Blood. 2015;125(8):1314-1324.

20. Weintraub J, Nkrumah FK, Evans CB, Tigelaar RE, Rosenberg YJ. Immunity to Plasmodium berghei yoelli in mice. II. Specific and nonspecific cellular and humoral responses during the course of infection. J Immunol. 1978;121(2):629.

21. Kim CC, Nelson CS, Wilson EB, Hou B, DeFranco AL, DeRisi JL. Splenic red pulp macrophages produce type I interferons as early sentinels of malaria infection but are dispensable for control. PLOS ONE. 2012;7(10):e48126.

22. Holmgren AM, Miller KD, Cavanaugh SE, Rall GF. Bst2/Tetherin is induced in neurons by type i interferon and viral infection but is dispensable for protection against neurotropic viral challenge. J Virol. 2015;89(21):11011-11018.

23. Blasius AL, Giurisato E, Cella M, Schreiber RD, Shaw AS, Colonna M. Bone marrow stromal cell antigen 2 is a specific marker of type I IFN-producing cells in the naive mouse, but a promiscuous cell surface antigen following IFN stimulation. J Immunol. 2006;177(5):3260-3265.

24. Spaulding E, et al. STING-licensed macrophages prime type I IFN production by plasmacytoid dendritic cells in the bone marrow during severe Plasmodium yoelii malaria. PLoS Pathog. 2016;12(10):e1005975

25. Lau L, Gray EE, Brunette RL, Stetson DB. DNA tumor virus oncogenes antagonize the cGAS-STING DNA-sensing pathway. Science. 2015;350(6260):568-571.

26. Taylor-Robinson AW, Phillips RS, Severn A, Moncada S, Liew FY. The role of TH1 and TH2 cells in a rodent malaria infection. Science. 1993;260(5116):1931-1934.

27. Hanson MC, et al. Nanoparticulate STING agonists are potent lymph node-targeted vaccine adjuvants. J Clin Invest. 2015;125(6):2532-2546.

28. Sebina I, et al. IFNAR1-signalling obstructs ICOS-mediated humoral immunity during non-lethal blood-stage plasmodium infection. PLoS Pathog. 2016;12(11):e1005999.

29. Lin JW, et al. The subcellular location of ovalbumin in Plasmodium berghei blood stages influences the magnitude of T-cell responses. Infect Immun. 2014;82(11):4654-4665.

30. Kurtulus S, Hildeman D. Assessment of CD4(+) and CD8 (+) T cell responses using MHC class I and II tetramers. Methods Mol Biol. 2013;979:71-79.

31. Oxenius A, Bachmann MF, Ashton-Rickardt PG, Tonegawa S, Zinkernagel RM, Hengartner H. Presentation of endogenous viral proteins in association with major histocompatibility complex class II: on the role of intracellular compartmentalization, invariant chain and the TAP transporter system. Eur J Immunol. 1995;25(12):3402-3411.

32. Tubo NJ, et al. Single naive CD4+ T cells from a diverse repertoire produce different effector cell types during infection. Cell. 2013;153(4):785-796.

33. Zander RA, et al. Type I interferons induce T regulatory 1 responses and restrict humoral immunity during experimental malaria. PLoS Pathog. 2016;12(10):e1005945.

34. Pérez-Mazliah D, et al. Disruption of IL-21 signaling affects T cell-B cell interactions and abrogates protective humoral immunity to malaria. PLoS Pathog. 2015;11(3):e1004715. 
35. Roberts DW, Rank RG, Weidanz WP, Finerty JF. Prevention of recrudescent malaria in nude mice by thymic grafting or by treatment with hyperimmune serum. Infect Immun. 1977;16(3):821-826.

36. Sze DM, Toellner KM, García de Vinuesa C, Taylor DR, MacLennan IC. Intrinsic constraint on plasmablast growth and extrinsic limits of plasma cell survival. J Exp Med. 2000;192(6):813-821.

37. Sanderson RD, Lalor P, Bernfield M. B lymphocytes express and lose syndecan at specific stages of differentiation. Cell Regul. 1989;1(1):27-35.

38. Keitany GJ, et al. Blood stage malaria disrupts humoral immunity to the pre-erythrocytic stage circumsporozoite protein. Cell Rep. 2016;17(12):3193-3205

39. Rono J, et al. Breadth of anti-merozoite antibody responses is associated with the genetic diversity of asymptomatic Plasmodium falciparum infections and protection against clinical malaria. Clin Infect Dis. 2013;57(10):1409-1416.

40. al-Yaman F, et al. Assessment of the role of naturally acquired antibody levels to Plasmodium falciparum merozoite surface protein-1 in protecting Papua New Guinean children from malaria morbidity. Am J Trop Med Hyg. 1996;54(5):443-448.

41. Eslava I, Payares G, Pernia BM, Holder AA, Spencer LM. Suppressive and additive effects in protection mediated by combinations of monoclonal antibodies specific for merozoite surface protein 1 of Plasmodium yoelii. Malar J. 2010;9:46.

42. Daly TM, Long CA. A recombinant 15-kilodalton carboxyl-terminal fragment of Plasmodium yoelii yoelii 17XL merozoite surface protein 1 induces a protective immune response in mice. Infect Immun. 1993;61(6):2462-2467.

43. Krishnamurty AT, et al. Somatically hypermutated Plasmodium-specific IgM(+) memory B cells are rapid, plastic, early responders upon malaria rechallenge. Immunity. 2016;45(2):402-414.

44. Ayieko C, et al. Changes in B cell populations and merozoite surface protein-1-specific memory B cell responses after prolonged absence of detectable P. falciparum infection. PLoS ONE. 2013;8(6):e67230.

45. Pape KA, Taylor JJ, Maul RW, Gearhart PJ, Jenkins MK. Different B cell populations mediate early and late memory during an endogenous immune response. Science. 2011;331(6021):1203-1207.

46. Langhorne J, Evans CB, Asofsky R, Taylor DW. Immunoglobulin isotype distribution of malaria-specific antibodies produced during infection with Plasmodium chabaudi adami and Plasmodium yoelii. Cell Immunol. 1984;87(2):452-461.

47. White WI, Evans CB, Taylor DW. Antimalarial antibodies of the immunoglobulin G2a isotype modulate parasitemias in mice infected with Plasmodium yoelii. Infect Immun. 1991;59(10):3547-3554.

48. Poholek AC, et al. In vivo regulation of Bcl6 and T follicular helper cell development. J Immunol. 2010;185(1):313-326.

49. Pepper M, Pagán AJ, Igyártó BZ, Taylor JJ, Jenkins MK. Opposing signals from the Bcl6 transcription factor and the interleukin-2 receptor generate T helper 1 central and effector memory cells. Immunity. 2011;35(4):583-595.

50. Kerfoot SM, et al. Germinal center B cell and T follicular helper cell development initiates in the interfollicular zone. Immunity. 2011;34(6):947-960

51. Yates JL, Racine R, McBride KM, Winslow GM. T cell-dependent IgM memory B cells generated during bacterial infection are required for IgG responses to antigen challenge. J Immunol. 2013;191(3):1240-1249.

52. Anderson SM, Tomayko MM, Ahuja A, Haberman AM, Shlomchik MJ. New markers for murine memory B cells that define mutated and unmutated subsets. J Exp Med. 2007;204(9):2103-2114.

53. Achtman AH, Khan M, MacLennan IC, Langhorne J. Plasmodium chabaudi chabaudi infection in mice induces strong B cell responses and striking but temporary changes in splenic cell distribution. J Immunol. 2003;171(1):317-324.

54. Carvalho LJ, Ferreira-da-Cruz MF, Daniel-Ribeiro CT, Pelajo-Machado M, Lenzi HL. Germinal center architecture disturbance during Plasmodium berghei ANKA infection in CBA mice. Malar J. 2007;6:59.

55. Urban BC, et al. Fatal Plasmodium falciparum malaria causes specific patterns of splenic architectural disorganization. Infect Immun. 2005;73(4):1986-1994.

56. Alves FA, et al. Splenic architecture disruption and parasite-induced splenocyte activation and anergy in Plasmodium falciparum-infected Saimiri sciureus monkeys. Malar J. 2015;14:128

57. Taylor DN, Wasi C, Bernard K. Chloroquine prophylaxis associated with a poor antibody response to human diploid cell rabies vaccine. Lancet. 1984;1(8391):1405.

58. Hou L, Block KE, Huang H. Artesunate abolishes germinal center B cells and inhibits autoimmune arthritis. PLoS ONE. 2014;9(8):e104762.

59. Vinuesa CG, Linterman MA, Yu D, MacLennan IC. Follicular helper T cells. Annu Rev Immunol. 2016;34:335-368.

60. Moser K, Tokoyoda K, Radbruch A, MacLennan I, Manz RA. Stromal niches, plasma cell differentiation and survival. Curr Opin Immunol. 2006;18(3):265-270.

61. Mesin L, Ersching J, Victora GD. Germinal center B cell dynamics. Immunity. 2016;45(3):471-482.

62. Corcoran LM, Tarlinton DM. Regulation of germinal center responses, memory B cells and plasma cell formation-an update. Curr Opin Immunol. 2016;39:59-67.

63. Roestenberg M, et al. Protection against a malaria challenge by sporozoite inoculation. N Engl J Med. 2009;361(5):468-477.

64. Wong KA, Zhou A, Rodriguez A. Protective immunity induced by daily bites from irradiated mosquitoes infected with Plasmodium yoelii. Parasite Immunol. 2008;30(9):482-486.

65. Li T, et al. Antitumor Activity of cGAMP via Stimulation of cGAS-cGAMP-STING-IRF3 mediated innate immune response. Sci Rep. 2016;6:19049

66. Gao D, et al. Activation of cyclic GMP-AMP synthase by self-DNA causes autoimmune diseases. Proc Natl Acad Sci USA. 2015;112(42):E5699-E5705.

67. Palomo J, et al. Type I interferons contribute to experimental cerebral malaria development in response to sporozoite or bloodstage Plasmodium berghei ANKA. Eur J Immunol. 2013;43(10):2683-2695.

68. Ball EA, et al. IFNAR1 controls progression to cerebral malaria in children and CD8+ T cell brain pathology in Plasmodium berghei-infected mice. J Immunol. 2013;190(10):5118-5127.

69. Haque A, et al. Type I IFN signaling in CD8- DCs impairs Th1-dependent malaria immunity. J Clin Invest. 2014;124(6):24832496.

70. Vigário AM, et al. Recombinant human IFN-alpha inhibits cerebral malaria and reduces parasite burden in mice. J Immunol. 2007;178(10):6416-6425. 
71. Morrell CN, et al. Beta interferon suppresses the development of experimental cerebral malaria. Infect Immun. 2011;79(4):1750-1758.

72. Voisine C, Mastelic B, Sponaas AM, Langhorne J. Classical CD11c+ dendritic cells, not plasmacytoid dendritic cells, induce T cell responses to Plasmodium chabaudi malaria. Int J Parasitol. 2010;40(6):711-719.

73. Haque A, et al. Type I interferons suppress CD4 $4^{+}$T-cell-dependent parasite control during blood-stage Plasmodium infection Eur J Immunol. 2011;41(9):2688-2698.

74. Edwards CL, et al. Spatiotemporal requirements for IRF7 in mediating type I IFN-dependent susceptibility to blood-stage Plasmodium infection. Eur J Immunol. 2015;45(1):130-141.

75. Wu J, et al. Strain-specific innate immune signaling pathways determine malaria parasitemia dynamics and host mortality. Proc Natl Acad Sci USA. 2014;111(4):E511-E520.

76. Cai B, Wu J, Yu X, Su XZ, Wang RF. FOSL1 inhibits type I interferon responses to malaria and viral infections by blocking TBK1 and TRAF3/TRIF interactions. MBio. 2017;8(1):e02161-16.

77. Stough JM, et al. Functional characteristics of the gut microbiome in C57BL/6 mice differentially susceptible to Plasmodium yoelii. Front Microbiol. 2016;7:1520.

78. Villarino NF, et al. Composition of the gut microbiota modulates the severity of malaria. Proc Natl Acad Sci USA. 2016;113(8):2235-2240.

79. Geurts N, et al. Insufficiently defined genetic background confounds phenotypes in transgenic studies as exemplified by malaria infection in Tlr9 knockout mice. PLoS One. 2011;6(11):e27131.

80. Spence PJ, et al. Vector transmission regulates immune control of Plasmodium virulence. Nature. 2013;498(7453):228-231.

81. Weiss L, Johnson J, Weidanz W. Mechanisms of splenic control of murine malaria: tissue culture studies of the erythropoietic interplay of spleen, bone marrow, and blood in lethal (strain 17XL) Plasmodium yoelii malaria in BALB/c mice. Am J Trop Med Hyg. 1989;41(2):135-143.

82. Cigel F, et al. Immunity to blood-stage murine malarial parasites is MHC class II dependent. Immunol Lett. 2003;89(2-3):243-249.

83. Brake DA, Long CA, Weidanz WP. Adoptive protection against Plasmodium chabaudi adami malaria in athymic nude mice by a cloned T cell line. J Immunol. 1988;140(6):1989-1993.

84. Grun JL, Weidanz WP. Immunity to Plasmodium chabaudi adami in the B-cell-deficient mouse. Nature. 1981;290(5802):143-145.

85. Steinman RM, Blumencranz SJ, Machtinger BG, Fried J, Cohn ZA. Mouse spleen lymphoblasts generated in vitro. Their replication and differentiation in vitro. JExp Med. 1978;147(2):297-315.

86. Weiss GE, et al. The Plasmodium falciparum-specific human memory B cell compartment expands gradually with repeated malaria infections. PLoS Pathog. 2010;6(5):e1000912.

87. Tran TM, et al. An intensive longitudinal cohort study of Malian children and adults reveals no evidence of acquired immunity to Plasmodium falciparum infection. Clin Infect Dis. 2013;57(1):40-47.

88. Weiss GE, et al. Atypical memory B cells are greatly expanded in individuals living in a malaria-endemic area. J Immunol. 2009;183(3):2176-2182.

89. Zhang TT, et al. Germinal center B cell development has distinctly regulated stages completed by disengagement from $\mathrm{T}$ cell help. Elife. 2017;6:e19552.

90. White MT, et al. Immunogenicity of the RTS,S/AS01 malaria vaccine and implications for duration of vaccine efficacy: secondary analysis of data from a phase 3 randomised controlled trial. Lancet Infect Dis. 2015;15(12):1450-1458.

91. Shanks GD, Hay SI, Bradley DJ. Malaria's indirect contribution to all-cause mortality in the Andaman Islands during the colonial era. Lancet Infect Dis. 2008;8(9):564-570.

92. Giglioli G. Changes in the pattern of mortality following the eradication of hyperendemic malaria from a highly susceptible community. Bull World Health Organ. 1972;46(2):181-202.

93. Gray EE, Treuting PM, Woodward JJ, Stetson DB. Cutting Edge: cGAS is required for lethal autoimmune disease in the Trex1-deficient mouse model of aicardi-goutières syndrome. J Immunol. 2015;195(5):1939-1943.

94. Gall A, et al. Autoimmunity initiates in nonhematopoietic cells and progresses via lymphocytes in an interferon-dependent autoimmune disease. Immunity. 2012;36(1):120-131

95. Moon JJ, et al. Tracking epitope-specific T cells. Nat Protoc. 2009;4(4):565-581.

96. Taylor JJ, Pape KA, Jenkins MK. A germinal center-independent pathway generates unswitched memory B cells early in the primary response. JExp Med. 2012;209(3):597-606. 